\title{
Inverse Scattering Transform for the Discrete Focusing Nonlinear Schrödinger Equation with Nonvanishing Boundary Conditions
}

\author{
Cornelis van der Mee \\ Dip. Matematica e Informatica, Università di Cagliari, Viale Merello 92 \\ 09123 Cagliari, Italy \\ cornelis110553@gmail.com
}

Received 24 October 2014

Accepted 3 February 2015

\begin{abstract}
In this article we develop the direct and inverse scattering theory of the Ablowitz-Ladik system with potentials having limits of equal positive modulus at infinity. In particular, we introduce fundamental eigensolutions, Jost solutions, and scattering coefficients, and study their properties. We also discuss the discrete eigenvalues and the corresponding norming constants. We then go on to derive the left Marchenko equations whose solutions solve the inverse scattering problem. We specify the time evolution of the scattering data to solve the initial-value problem of the corresponding integrable discrete nonlinear Schrödinger equation. The one-soliton solution is also discussed.
\end{abstract}

Keywords: Inverse Scattering transform; Ablowitz-Ladik system; Discrete focusing Nonlinear Schrödinger Equation.

2000 Mathematics Subject Classification: 37K15, 35Q55, 39A70

\section{Introduction}

The nonlinear Schrödinger (NLS) equation

$$
i q_{t}=q_{x x}+2 \sigma|q|^{2} q
$$

is a universal model for weakly nonlinear dispersive waves arising in many different contexts. The initial-value problem for (1.1) on the real line can be solved via the inverse scattering transform (IST) method, both in the focusing $(\sigma=+1)$ and in the defocusing $(\sigma=-1)$ case. Most research involves solutions vanishing as $x \rightarrow \pm \infty[5,6,16,26,31,33]$. Recently there has been much interest in solutions nonvanishing as $x \rightarrow \pm \infty[8,10,11]$.

In this article we study the IST for the focusing NLS equation which is discrete in position and continuous in time, obtained by applying forward differencing to the focusing Zakharov-Shabat system

$$
v_{x}=\left(\begin{array}{cc}
-i k & q \\
r & i k
\end{array}\right) v
$$

accompanying the focusing NLS equation as the linear eigenvalue problem under the IST. For potentials vanishing as $n \rightarrow \pm \infty$ this discretization was first formulated by Ablowitz and Ladik [2-5], 
leading to the corresponding integrable discrete nonlinear Schrödinger (IDNLS) equation

$$
i \frac{d}{d \tau} q_{n}=\frac{q_{n+1}-2 q_{n}+q_{n-1}}{h^{2}}+\left(q_{n+1}+q_{n-1}\right)\left|q_{n}\right|^{2} .
$$

Equation (1.3) was generalized to the defocusing case with small nonvanishing boundary conditions $[1,32]$. Equation (1.3) with vanishing boundary values, where $q_{n}$ is a rectangular matrix, has been studied in $[5,12,13,17,18]$.

As a discretization of the nonlinear Schrödinger (NLS) equation (1.1), the IDNLS equation (1.3) has important applications to electromagnetic wave propagation in nonlinear media [33], surface waves on deep waters [33], and signal propagation in optical fibers [19,20]. On its own behalf, the IDNLS equation has applications to Heisenberg spin chains [22,27], self-trapping on a dimer [23], anharmonic lattices [29], the dynamics of a discrete curve on an ultraspherical surface [15], the dynamics of triangulations of surfaces [21], and Hamiltonian flows [24, 28].

The scaling transformations $Q_{n}=h q_{n}, R_{n}=h r_{n}, z=1-i h k+O\left(h^{2}\right), z^{-1}=1+i h k+O\left(h^{2}\right)$, and $n h \leftarrow x$ convert the Zakharov-Shabat system into the Ablowitz-Ladik system

$$
v_{n+1}=\left(\boldsymbol{Z}+\boldsymbol{q}_{n}\right) v_{n},
$$

where the position $n \in \mathbb{Z}$ and

$$
\boldsymbol{Z}=\left(\begin{array}{cc}
z & 0 \\
0 & z^{-1}
\end{array}\right), \quad \boldsymbol{q}_{n}=\left(\begin{array}{cc}
0 & q_{n} \\
r_{n} & 0
\end{array}\right) .
$$

Using a suitable AKNS pair [5,31] and the additional scaling transformation $t=h^{2} \tau$, we obtain (1.3) from (1.1).

Generalizing the existing literature [5, Ch. 3], we make the following assumption on the potential $\left\{\boldsymbol{q}_{n}\right\}_{n \in \mathbb{Z}}$ :

$$
\left(\boldsymbol{H}_{s}\right) \quad \sum_{n=0}^{\infty}(1+|n|)^{s}\left\{\left\|\boldsymbol{q}_{n}-\boldsymbol{q}_{r}\right\|+\left\|\boldsymbol{q}_{-n}-\boldsymbol{q}_{l}\right\|\right\}<+\infty,
$$

where $s=0,1,2$. Here

$$
A=\left\|\boldsymbol{q}_{r}\right\|=\left\|\boldsymbol{q}_{l}\right\|>0 .
$$

Unless stated otherwise, the norm of a square matrix is its largest singular value. Throughout this article we restrict ourselves to the focusing case, where $\boldsymbol{q}_{n}^{\dagger}=-\boldsymbol{q}_{n}$ and hence $\boldsymbol{q}_{r / l}^{\dagger}=-\boldsymbol{q}_{r / l}$ and the dagger denotes the matrix conjugate transpose and the asterisk the complex conjugate. In the focusing case we have $A=\left|q_{r}\right|=\left|q_{l}\right|>0$.

The scalar IDNLS equation (1.3) was first studied by Ablowitz and Ladik [2-4] using the inverse scattering transform (IST) method. Early work on the Ablowitz-Ladik system, where $q_{n}$ is a rectangular matrix vanishing as $n \rightarrow \pm \infty$, was done by Gerdjikov and Ivanov [17,18]. A comprehensive theory of these more general systems was developed by Ablowitz, Prinari, and Trubatch [5, Ch. 5] and by Tsuchida, Ujino, and Wadati [30]. Tsuchida et al. [30] have also derived the $N$-soliton and breather solutions to (1.1) in terms of solutions to $N \times N$ linear systems [30, Eq. (3.43)]. Some of these breather solutions were constructed before by using the Hirota method [9]. An extensive class of exact solutions of the matrix IDNLS equation with vanishing boundary conditions was derived by the matrix triplet method by Demontis and Van der Mee [12-14]. 
Let us discuss the contents of this article. In Section 2 we prove the existence of the fundamental eigensolutions and evaluate their determinants. In Sections 3 and 4 we introduce the conformal transformation of the spectral variable $z$ which allows us to define and study the Jost solutions and scattering coefficients and to formulate the inverse scattering problem. Section 5 is devoted to the socalled triangular representations of fundamental eigensolutions and Jost solutions. In Section 6 we derive the left Marchenko equations required to solve the inverse scattering problem and compute the one-soliton solution. The time evolution of the scattering data required to solve the initialvalue problem for the IDNLS equation (1.3) by means of the IST will be derived in Section 7. In Appendices A-D we derive the discrete Gronwall inequality, study the asymptotic behavior of the conformal mapping $\zeta(z)$ near $z=0$ and $\zeta=\infty$, determine the coefficients of the power series expansion of the conformal mapping in terms of Jacobi polynomials, and detail the derivation of the time evolution of the scattering data. Throughout Sections 2-6 we do not write the $\tau$-dependence of the various quantities explicitly, except in the one-soliton solution.

\section{Fundamental eigensolutions}

In this section we introduce the fundamental eigensolutions and compute their determinants. We also interrelate the fundamental eigensolutions.

Let us briefly discuss the spectral properties of Eq. (1.4) for nonvanishing boundary conditions and analyze the matrix $\left(1+A^{2}\right)^{-1 / 2}\left[\boldsymbol{Z}+\boldsymbol{q}_{r / l}\right]$ of determinant 1 . Letting $\Sigma$ stand for the set of those $z \in \mathbb{C}$ for which the eigenvalues of $\left(1+A^{2}\right)^{-1 / 2}\left[\boldsymbol{Z}+\boldsymbol{q}_{r / l}\right]$ belong to the unit circle $\mathbb{T}$, we obtain

$$
\Sigma=\mathbb{T} \cup\left\{z \in \mathbb{R}:\left|z-\sqrt{1+A^{2}}\right| \leq A\right\} \cup\left\{z \in \mathbb{R}:\left|z+\sqrt{1+A^{2}}\right| \leq A\right\}
$$

Then the essential spectrum of (1.4) coincides with $\Sigma$.

It is easily verified that

$$
\left(\frac{\boldsymbol{Z}+\boldsymbol{q}_{r / l}}{\sqrt{1+A^{2}}}\right)^{-1}=\frac{\boldsymbol{Z}^{-1}-\boldsymbol{q}_{r / l}}{\sqrt{1+A^{2}}} .
$$

For $z \in \mathbb{T}$ the matrix $\left(1+A^{2}\right)^{-1 / 2}\left[\boldsymbol{Z}+\boldsymbol{q}_{r / l}\right]$ is unitary. Moreover,

$$
\left\|\left(\frac{\boldsymbol{Z}+\boldsymbol{q}_{r / l}}{\sqrt{1+A^{2}}}\right)^{n}\right\| \leq \begin{cases}C(z), & -A<z \pm \sqrt{1+A^{2}}<A, \\ \hat{C}(1+|n|), & -A \leq z \pm \sqrt{1+A^{2}} \leq A,\end{cases}
$$

where $C(z)$ is the condition number of the transformation diagonalizing $\left(1+A^{2}\right)^{-1 / 2}\left[\boldsymbol{Z}+\boldsymbol{q}_{r / l}\right]$ and $\hat{C}$ is some constant.

The following result establishes the existence of the fundamental eigensolutions $\tilde{\Psi}_{n}(z)$ and $\tilde{\Phi}_{n}(z)$ to the discrete Zakharov-Shabat system (1.4) under the hypothesis $\left(\boldsymbol{H}_{0}\right)$.

Theorem 2.1. Assume the hypothesis $\left(\boldsymbol{H}_{0}\right)$ is true. Then for any $z \in \Sigma$ which is not one of the four points $\pm \sqrt{1+A^{2}} \pm A$, there exists unique solutions $\tilde{\Psi}_{n}(z)$ and $\tilde{\Phi}_{n}(z)$ to (1.4) satisfying

$$
\begin{aligned}
\tilde{\Psi}_{n}(z) & =\left(\boldsymbol{Z}+\boldsymbol{q}_{r}\right)^{n}\left[I_{2}+o(1)\right], & & n \rightarrow+\infty, \\
\tilde{\Phi}_{n}(z) & =\left(\boldsymbol{Z}+\boldsymbol{q}_{l}\right)^{n}\left[I_{2}+o(1)\right], & & n \rightarrow-\infty .
\end{aligned}
$$


These solutions satisfy the discrete Volterra equations

$$
\begin{aligned}
& \tilde{\Psi}_{n}(z)=\left(\boldsymbol{Z}+\boldsymbol{q}_{n}\right)^{-1}\left\{\left(\boldsymbol{Z}+\boldsymbol{q}_{r}\right)^{n+1}-\sum_{s=n+1}^{\infty}\left(\boldsymbol{Z}+\boldsymbol{q}_{r}\right)^{n-s}\left(\boldsymbol{q}_{s}-\boldsymbol{q}_{r}\right) \tilde{\Psi}_{s}(z)\right\}, \\
& \tilde{\Phi}_{n}(z)=\left(\boldsymbol{Z}+\boldsymbol{q}_{l}\right)^{n}+\sum_{s=-\infty}^{n-1}\left(\boldsymbol{Z}+\boldsymbol{q}_{l}\right)^{n-s-1}\left(\boldsymbol{q}_{s}-\boldsymbol{q}_{l}\right) \tilde{\Phi}_{s}(z) .
\end{aligned}
$$

Under the hypothesis $\left(\boldsymbol{H}_{1}\right)$ the result is true for any $z \in \Sigma$.

Proof. Substituting $v_{n}=\tilde{\Psi}_{n}(z)=\left(\boldsymbol{Z}+\boldsymbol{q}_{r}\right)^{n} W_{n}$ into (1.4) and assuming that $W_{n} \rightarrow I_{2}$ as $n \rightarrow+\infty$, we arrive at the equality

$$
W_{n+1}-W_{n}=\left(\boldsymbol{Z}+\boldsymbol{q}_{r}\right)^{-(n+1)}\left(\boldsymbol{q}_{n}-\boldsymbol{q}_{r}\right)\left(\boldsymbol{Z}+\boldsymbol{q}_{r}\right)^{n} W_{n} .
$$

Replacing $n$ by $n+j$ for $j=0,1, \cdots, p-1$ and adding the resulting equations we get

$$
W_{n+p}-W_{n}=\sum_{j=0}^{p-1}\left(\boldsymbol{Z}+\boldsymbol{q}_{r}\right)^{-(n+j+1)}\left(\boldsymbol{q}_{n+j}-\boldsymbol{q}_{r}\right)\left(\boldsymbol{Z}+\boldsymbol{q}_{r}\right)^{n+j} W_{n+j} .
$$

Using (2.3) we estimate for $z \in \Sigma$ which is not one of the four points $\pm \sqrt{1+A^{2}} \pm A$,

$$
\left\|\left(\boldsymbol{Z}+\boldsymbol{q}_{r}\right)^{-(n+j+1)}\left(\boldsymbol{q}_{n+j}-\boldsymbol{q}_{r}\right)\left(\boldsymbol{Z}+\boldsymbol{q}_{r}\right)^{n+j} W_{n+j}\right\| \leq \frac{C_{r}(z)^{2}}{\sqrt{1+A_{r}^{2}}}\left\|Q_{n}-Q_{r}\right\|\left\|W_{n}\right\| .
$$

Letting $p \rightarrow+\infty$, we obtain

$$
W_{n}=I_{2}-\sum_{j=0}^{\infty}\left(\boldsymbol{Z}+\boldsymbol{q}_{r}\right)^{-(n+j+1)}\left(\boldsymbol{q}_{n+j}-\boldsymbol{q}_{r}\right)\left(\boldsymbol{Z}+\boldsymbol{q}_{r}\right)^{n+j} W_{n+j} .
$$

Hence,

$$
\tilde{\Psi}_{n}(z)=\left(\boldsymbol{Z}+\boldsymbol{q}_{r}\right)^{n}-\sum_{j=0}^{\infty}\left(\boldsymbol{Z}+\boldsymbol{q}_{r}\right)^{-(j+1)}\left(\boldsymbol{q}_{n+j}-\boldsymbol{q}_{r}\right) \tilde{\Psi}_{n+j}(z) .
$$

Reshuffling the latter equation, we get

$$
\left(\boldsymbol{Z}+\boldsymbol{q}_{r}\right)^{-1}\left(\boldsymbol{Z}+\boldsymbol{q}_{n}\right) \tilde{\Psi}_{n}(z)=\left(\boldsymbol{Z}+\boldsymbol{q}_{r}\right)^{n}-\sum_{j=1}^{\infty}\left(\boldsymbol{Z}+\boldsymbol{q}_{r}\right)^{-(j+1)}\left(\boldsymbol{q}_{n+j}-\boldsymbol{q}_{r}\right) \tilde{\Psi}_{n+j}(z)
$$

which implies (2.5a). Letting $B=\sup _{n \in \mathbb{Z}}\left\|\left(\boldsymbol{Z}+\boldsymbol{q}_{n}\right)^{-1}\left(\boldsymbol{Z}+\boldsymbol{q}_{r}\right)\right\|$, we apply Proposition A.1 to obtain

$$
\left\|\tilde{\Psi}_{n}(z)\right\| \leq B C(z)\left(1+A^{2}\right)^{\frac{n}{2}} \exp \left[B C(z) \sum_{j=n+1}^{\infty}\left[1+A^{2}\right]^{-\frac{j+1}{2}}\left\|\boldsymbol{q}_{n+j}-\boldsymbol{q}_{r}\right\|\right] .
$$

For $z \in \Sigma$ and under the hypothesis $\left(\boldsymbol{H}_{1}\right)$ we obtain instead

$$
\left\|\tilde{\Psi}_{n}(z)\right\| \leq B \hat{C}\left(1+A^{2}\right)^{\frac{n}{2}} \exp \left[B \hat{C} \sum_{j=n+1}^{\infty}\left[1+A^{2}\right]^{-\frac{j+1}{2}}[2+|j|]\left\|\boldsymbol{q}_{n+j}-\boldsymbol{q}_{r}\right\|\right] .
$$

Next, substitute $v_{n}=\tilde{\Phi}_{n}(z)=\left(\boldsymbol{Z}+\boldsymbol{q}_{l}\right)^{n} W_{n}$ into (1.4), assuming that $W_{n} \rightarrow I_{2}$ as $n \rightarrow-\infty$. Then we obtain (2.6) with $\boldsymbol{q}_{r}$ replaced by $\boldsymbol{q}_{l}$. Replacing in this modified (2.6) $n$ by $n-1-j$ for $j=$ 
$0,1, \ldots, p-1$ and adding the resulting equations we get

$$
W_{n}-W_{n-p}=\sum_{j=0}^{p-1}\left(\boldsymbol{Z}+\boldsymbol{q}_{l}\right)^{-(n-j)}\left(\boldsymbol{q}_{n-j-1}-\boldsymbol{q}_{l}\right)\left(\boldsymbol{Z}+\boldsymbol{q}_{l}\right)^{n-j-1} W_{n-j-1} .
$$

Letting $p \rightarrow+\infty$, we obtain

$$
W_{n}=I_{2}+\sum_{j=0}^{\infty}\left(\boldsymbol{Z}+\boldsymbol{q}_{l}\right)^{-(n-j)}\left(\boldsymbol{q}_{n-j-1}-\boldsymbol{q}_{l}\right)\left(\boldsymbol{Z}+\boldsymbol{q}_{l}\right)^{n-j-1} W_{n-j-1},
$$

where

$$
\begin{aligned}
& \left\|\left(\boldsymbol{Z}+\boldsymbol{q}_{l}\right)^{-(n-j)}\left(\boldsymbol{q}_{n-j-1}-\boldsymbol{q}_{l}\right)\left(\boldsymbol{Z}+\boldsymbol{q}_{l}\right)^{n-j-1} W_{n-j-1}\right\| \\
& \leq \frac{C_{l}(z)^{2}}{\sqrt{1+A^{2}}} \sum_{j=0}^{\infty}\left\|\boldsymbol{q}_{n-j-1}-\boldsymbol{q}_{l}\right\|\left\|W_{n-j-1}\right\| .
\end{aligned}
$$

Hence,

$$
\tilde{\Phi}_{n}(z)=\left(\boldsymbol{Z}+\boldsymbol{q}_{l}\right)^{n}+\sum_{j=0}^{\infty}\left(\boldsymbol{Z}+\boldsymbol{q}_{l}\right)^{j}\left(\boldsymbol{q}_{n-j-1}-\boldsymbol{q}_{l}\right) \tilde{\Phi}_{n-j-1}(z),
$$

which implies (2.5b). Thus, by Proposition A.2 we have

$$
\left\|\tilde{\Phi}_{n}(z)\right\| \leq C(z)\left(1+A^{2}\right)^{\frac{n-1}{2}} \exp \left[\sum_{s=-\infty}^{n-1} C(z)\left[1+A^{2}\right]^{n-s-1}\left\|Q_{s}-Q_{l}\right\|\right] .
$$

For $z \in \Sigma$ we modify the estimate to

$$
\begin{aligned}
\left\|\tilde{\Phi}_{n}(z)\right\| & \leq \hat{C}[2+|n|]\left(1+A^{2}\right)^{\frac{n-1}{2}} \times \\
& \times \exp \left[\sum_{s=-\infty}^{n-1} \hat{C}[2+|n-s|]\left[1+A^{2}\right]^{n-s-1}\left\|Q_{s}-Q_{l}\right\|\right],
\end{aligned}
$$

which completes the proof.

We now compute the determinants of the fundamental eigensolutions.

Proposition 2.1. Assume the hypothesis $\left(\boldsymbol{H}_{0}\right)$ is true. Then for any $z \in \Sigma$ which is not one of the points $\pm \sqrt{1+A^{2}} \pm A$, we have

$$
\begin{aligned}
& \operatorname{det} \tilde{\Psi}_{n}(z)=\left(1+A^{2}\right)^{n} \prod_{s=n}^{\infty} \frac{1+A^{2}}{1+\left|q_{s}\right|^{2}} \\
& \operatorname{det} \tilde{\Phi}_{n}(z)=\left(1+A^{2}\right)^{n} \prod_{s=-\infty}^{n-1} \frac{1+\left|q_{s}\right|^{2}}{1+A^{2}} .
\end{aligned}
$$

Under the hypothesis $\left(\boldsymbol{H}_{1}\right)$ the result is true for any $z \in \Sigma$.

Proof. The infinite products appearing in (2.8) converge absolutely and represent nonzero numbers, because of the estimate

$$
\left|\frac{1+\left|q_{s}\right|^{2}}{1+A^{2}}-1\right|=\frac{\left.|| q_{s}\right|^{2}-A^{2} \mid}{1+A^{2}} \leq\left|q_{s}-q_{r / l}\right|\left(\left|q_{s}-q_{r / l}\right|+2 A\right)
$$

and the hypothesis $\left(\boldsymbol{H}_{0}\right)$. 
Applying the determinant to (1.4) with $n$ replaced by $s=n, n+1, \ldots, n+p-1$ and multiplying the $p$ resulting ratios, we get

$$
\frac{\operatorname{det}\left[\left(\boldsymbol{Z}+\boldsymbol{q}_{r}\right)^{-n} \tilde{\Psi}_{n}(z)\right]}{\operatorname{det}\left[\left(\boldsymbol{Z}+\boldsymbol{q}_{r}\right)^{-(n+p)} \tilde{\Psi}_{n+p}(z)\right]}=\prod_{s=n}^{n+p-1} \frac{1+A^{2}}{1+\left|q_{s}\right|^{2}} .
$$

Taking the limit as $p \rightarrow+\infty$ we arrive at (2.8a). Analogously,

$$
\frac{\operatorname{det}\left[\left(\boldsymbol{Z}+\boldsymbol{q}_{r}\right)^{-n} \tilde{\Phi}_{n}(z)\right]}{\operatorname{det}\left[\left(\boldsymbol{Z}+\boldsymbol{q}_{r}\right)^{-(n-p)} \tilde{\Phi}_{n-p}(z)\right]}=\prod_{s=n-p}^{n-1} \frac{1+\left|q_{s}\right|^{2}}{1+A^{2}} .
$$

Taking the limit as $p \rightarrow+\infty$ we arrive at (2.8b).

Since the difference equation (1.4) is first order, we easily verify that

$$
\tilde{\Phi}_{n}(z)=\tilde{\Psi}_{n}(z) \tilde{\Psi}_{0}(z)^{-1} \tilde{\Phi}_{0}(z), \quad \tilde{\Psi}_{n}(z)=\tilde{\Phi}_{n}(z) \tilde{\Phi}_{0}(z)^{-1} \tilde{\Psi}_{0}(z),
$$

where $z \in \Sigma$. Put

$$
\begin{aligned}
& \boldsymbol{B}_{l}(z)=\tilde{\Psi}_{0}(z)^{-1} \tilde{\Phi}_{0}(z), \\
& \boldsymbol{B}_{r}(z)=\tilde{\Phi}_{0}(z)^{-1} \tilde{\Psi}_{0}(z),
\end{aligned}
$$

where $z \in \Sigma$. Then

$$
\begin{array}{llrl}
\tilde{\Psi}_{n}(z) & =\left(\boldsymbol{Z}+\boldsymbol{q}_{l}\right)^{n} \boldsymbol{B}_{r}(z)+o(1), & & n \rightarrow-\infty, \\
\tilde{\Phi}_{n}(z) & =\left(\boldsymbol{Z}+\boldsymbol{q}_{r}\right)^{n} \boldsymbol{B}_{l}(z)+o(1), & & n \rightarrow+\infty .
\end{array}
$$

Further, as a result of Proposition 2.1, for any $z \in \Sigma$ we get

$$
\operatorname{det} \boldsymbol{B}_{l}(z)=\frac{1}{\operatorname{det} \boldsymbol{B}_{r}(z)}=\prod_{s=-\infty}^{\infty} \frac{1+\left|q_{s}\right|^{2}}{1+A^{2}} .
$$

\section{Conformal mappings and Jost solutions}

The matrix $\left(1+A^{2}\right)^{-1 / 2}\left[\boldsymbol{Z}+\boldsymbol{q}_{r / l}\right]$ has the two eigenvalues

$$
\zeta^{ \pm}=\frac{z+z^{-1}}{2 \sqrt{1+A^{2}}} \pm i \sqrt{1-\left(\frac{z+z^{-1}}{2 \sqrt{1+A^{2}}}\right)^{2}}
$$

with product 1 . Then $\zeta^{ \pm}$belong to the unit circle $\mathbb{T}$ iff $z \in \Sigma$.

a. Conformal mappings. The conformal mappings $\zeta^{ \pm}(z)$ can be viewed as the composition of the conformal mappings

$$
w=\frac{z+z^{-1}}{2 \sqrt{1+A^{2}}}
$$

and

$$
\zeta^{ \pm}=w \pm \sqrt{w^{2}-1}
$$

The map (3.2) turns $\Sigma$ into $[-1,1]$. The set $\Sigma$ divides the complex $z$-plane into a bounded open region $\mathbb{K}^{+}$and an unbounded open region $\mathbb{K}^{-}$(transformed into each other by applying $z \mapsto z^{-1}$ ) which are both mapped onto the complex $w$-plane cut along $[-1,1]$. 


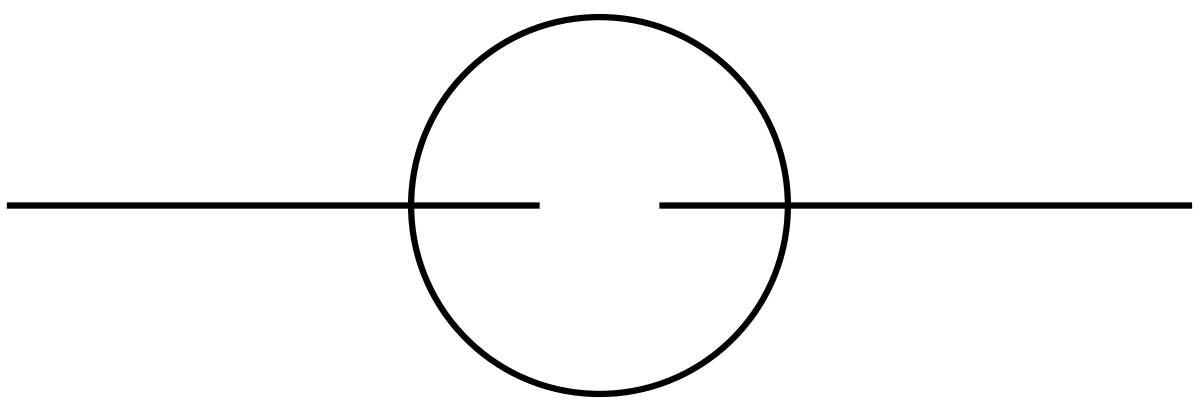

Fig. 1. Continuous spectrum $z \in \Sigma$. The conformal transformation $\zeta(z)$ maps the interior region $\mathbb{K}^{+}$to the open unit $\zeta$-disk $\Lambda^{+}$and the exterior region $\mathbb{K}^{-}$to the exterior unit $\zeta$-disk $\Lambda^{-}$. We have selected $A=\sqrt{2}$.

The map (3.3) is assumed to have its branch cut along the interval $[-1,1]$ and to satisfy $\zeta^{+} \sim 2 w$ as $w \rightarrow \infty$, while $\zeta^{+}(w) \zeta^{-}(w)=1$. Then for $|w|>1$ we have the absolutely convergent series

$$
\zeta^{+}(w)=2 w-\sum_{m=1}^{\infty} \frac{(2 m-1) ! !}{2^{m} m !} w^{-(2 m-1)}, \quad \zeta^{-}(w)=\sum_{m=1}^{\infty} \frac{(2 m-1) ! !}{2^{m} m !} w^{-(2 m-1)} .
$$

Its inverse map is given by

$$
w=\frac{1}{2}\left(\zeta^{ \pm}+\frac{1}{\zeta^{ \pm}}\right)
$$

The map $\zeta^{+}(w)$ is an odd function mapping the half-lines $[1,+\infty)$ and $(-\infty,-1]$ onto themselves, the positive and negative imaginary axes onto the respective half-lines $[i,+i \infty)$ and $(-i \infty,-i]$, the upper and lower edges of the branch cut onto the upper and lower halves of the unit circle centered about the origin, and therefore the complex plane cut along the interval $[-1,1]$ onto the exterior region of the unit circle centered about the origin. This map transforms each quadrant onto the part of the quadrant lying outside the unit circle centered about the origin.

The map $\zeta^{-}(w)$ is an odd function mapping the half-lines $[1,+\infty)$ and $(-\infty,-1]$ onto $(0,1]$ and $[-1,0)$, the positive and negative imaginary axes onto the respective half-lines $(i 0, i]$ and $(-i 0,-i]$, the upper and lower edges of the branch cut onto the lower and upper halves of the unit circle centered about the origin, and therefore the complex plane cut along the interval $[-1,1]$ onto the interior region of the unit circle centered about the origin. This map transforms each quadrant onto the part of the complex conjugate quadrant lying inside the unit circle centered about the origin.

We now define [cf. Fig. 1]

$$
\zeta(z)= \begin{cases}\zeta^{-}[w(z)], & z \in \mathbb{K}^{+} \\ \zeta^{+}[w(z)], & z \in \mathbb{K}^{-}\end{cases}
$$

Then the unit $\zeta$-circle acts as a common boundary of the open unit interior disk $\Lambda^{+}$(including zero) and the open unit exterior disk $\Lambda^{-}$(including infinity). The function $\zeta(z)$ maps $\mathbb{K}^{ \pm}$conformally onto $\Lambda^{ \pm}$and has the following four symmetry properties: (i) parity symmetry $\zeta(-z)=-\zeta(z)$, (ii) inversion symmetry $\zeta\left(z^{-1}\right)=\zeta(z)^{-1}$, (iii) real conjugation symmetry $\zeta\left(z^{*}\right)=\zeta(z)^{*}$, and (iv) circle conjugation symmetry $\zeta\left(z^{*-1}\right)=\zeta(z)^{*-1}$. 
b. Definition of Jost solutions. Let us find a matrix $W_{r / l}(z)$ such that

$$
\left(1+A^{2}\right)^{-\frac{1}{2}}\left[\mathbf{Z}+\boldsymbol{q}_{r / l}\right] W_{r / l}(z)=W_{r / l}(z) \mathscr{Z},
$$

where $\mathscr{Z}=\operatorname{diag}\left(\zeta, \zeta^{-1}\right)$ and $\zeta(z)$ is defined by (3.4). A convenient choice is the following

$$
W_{r / l}(z)=I_{2}+\gamma(z) \sigma_{3} \boldsymbol{q}_{r / l},
$$

where

$$
\begin{aligned}
\gamma(z) & =\left[z^{-1}-\zeta \sqrt{1+A^{2}}\right]^{-1}=\left[\zeta^{-1} \sqrt{1+A^{2}}-z\right]^{-1}, \\
A^{2} \gamma(z) & =\zeta \sqrt{1+A^{2}}-z=z^{-1}-\zeta^{-1} \sqrt{1+A^{2}}
\end{aligned}
$$

are piecewise analytic functions in $\zeta \in \mathbb{C} \cup\{\infty\}$ with a jump condition at each point $\zeta \in \mathbb{T}$ [cf. Appendix B]. The determinant of $W_{r / l}(z)$ is given by

$$
\begin{aligned}
1-A^{2} \gamma(z)^{2} & =\frac{\varepsilon}{A}\left[(\zeta \mp 1) \sqrt{1+A^{2}} \pm \sqrt{1+A^{2}}+\varepsilon A-z\right] \\
& \pm \frac{1}{A}\left[z^{-1} \mp \sqrt{1-A^{2}}+\varepsilon A-\left(\zeta^{-1} \mp 1\right) \sqrt{1+A^{2}}\right]
\end{aligned}
$$

where $\varepsilon= \pm 1$ and the \pm signs represent four situations. As a result, $\operatorname{det} W_{r / l}(z)=0$ iff $z$ is one of the four points $\pm \sqrt{1+A^{2}}+\varepsilon A$, where $\varepsilon= \pm 1$. Finally, we have the symmetry relations

$$
\begin{aligned}
\gamma(-z) & =-\gamma(z), & \gamma\left(z^{-1}\right) & =-\gamma(z), \\
\gamma\left(z^{*}\right)^{*} & =\gamma(z), & \gamma\left(z^{*-1}\right)^{*} & =-\gamma(z) .
\end{aligned}
$$

As a result,

$$
\begin{aligned}
W_{r / l}(-z) & =\sigma_{3} W_{r / l}(z) \sigma_{3}, \\
W_{r / l}\left(z^{-1}\right) & =\sigma_{2} e^{-i \theta_{r / l} \sigma_{3}} W_{r / l}(z) e^{i \theta_{r / l} \sigma_{3}} \sigma_{2}, \\
W_{r / l}\left(z^{*}\right)^{\dagger} & =\sigma_{1} e^{-i \theta_{r / l} \sigma_{3}} W_{r / l}(z) e^{i \theta_{r / l} \sigma_{3}} \sigma_{1}, \\
W_{r / l}\left(z^{*-1}\right)^{\dagger} & =\sigma_{3} W_{r / l}(z) \sigma_{3}=\left(1-A^{2} \gamma(z)^{2}\right) W_{r / l}(z)^{-1},
\end{aligned}
$$

where $q_{r / l}=A e^{i \theta_{r / l}}$ and $\sigma_{1}=\left(\begin{array}{ll}0 & 1 \\ 1 & 0\end{array}\right), \sigma_{2}=\left(\begin{array}{cc}0 & -i \\ i & 0\end{array}\right)$, and $\sigma_{3}=\left(\begin{array}{cc}1 & 0 \\ 0 & -1\end{array}\right)$ are the Pauli matrices.

Distinguishing the two limiting values of $\gamma(z)$ for $\zeta \in \mathbb{T}$, we now define the Jost matrices $\Psi_{n}(z)$ and $\Phi_{n}(z)$ and their columns, the Jost solutions, by

$$
\begin{aligned}
& \Psi_{n}(z)=\left(\bar{\psi}_{n}(z) \psi_{n}(z)\right)=\tilde{\Psi}_{n}(z) W_{r}(z), \\
& \Phi_{n}(z)=\left(\phi_{n}(z) \bar{\phi}_{n}(z)\right)=\tilde{\Phi}_{n}(z) W_{l}(z) .
\end{aligned}
$$

Thus the Jost solutions are defined for $z \in \partial \mathbb{K}^{+} \cup \partial \mathbb{K}^{-}$, where $\partial \mathbb{K}^{ \pm}$are the boundaries of $\mathbb{K}^{ \pm}$as analytic manifolds. They satisfy the asymptotic conditions [cf. (2.4) and (3.5)]

$$
\begin{array}{ll}
\Psi_{n}(z)=\left(1+A^{2}\right)^{\frac{n}{2}} W_{r}(z) \mathscr{Z}^{n}\left[I_{2}+o(1)\right], & n \rightarrow+\infty, \\
\Phi_{n}(z)=\left(1+A^{2}\right)^{\frac{n}{2}} W_{l}(z) \mathscr{Z}^{n}\left[I_{2}+o(1)\right], & n \rightarrow-\infty .
\end{array}
$$


c. Symmetry relations. By parity symmetry we mean the easily verified property that

$$
\tilde{V}_{n}(z)=(-1)^{n} \sigma_{3} V_{n}(-z) \sigma_{3}
$$

is a square matrix solution to (1.4) iff $V_{n}(z)$ is a square matrix solution to (1.4). Since

$$
(-1)^{n} \sigma_{3}\left(-\boldsymbol{Z}+\boldsymbol{q}_{r / l}\right)^{n} \sigma_{3}=\sigma_{3}\left(\boldsymbol{Z}-\boldsymbol{q}_{r / l}\right)^{n} \sigma_{3}=\left(\boldsymbol{Z}+\boldsymbol{q}_{r / l}\right)^{n},
$$

we obtain the following symmetry properties of the fundamental eigensolutions:

$$
(-1)^{n} \sigma_{3} \tilde{\Psi}_{n}(-z) \sigma_{3}=\tilde{\Psi}_{n}(z), \quad(-1)^{n} \sigma_{3} \tilde{\Phi}_{n}(-z) \sigma_{3}=\tilde{\Phi}_{n}(z) .
$$

Thus, using (3.13) and (2.9), we have

$$
\boldsymbol{B}_{l}(-z)=\sigma_{3} \boldsymbol{B}_{l}(z) \sigma_{3}, \quad \boldsymbol{B}_{r}(-z)=\sigma_{3} \boldsymbol{B}_{r}(z) \sigma_{3} .
$$

Using (3.9a) and (3.14) we get the parity symmetry relations

$$
\begin{aligned}
(-1)^{n} \sigma_{3}\left(\bar{\Psi}_{n}(-z) \psi_{n}(-z)\right) \sigma_{3} & =\left(\bar{\psi}_{n}(z) \psi_{n}(z)\right), \\
(-1)^{n} \sigma_{3}\left(\phi_{n}(-z) \bar{\phi}_{n}(-z)\right) \sigma_{3} & =\left(\phi_{n}(z) \bar{\phi}_{n}(z)\right) .
\end{aligned}
$$

Equation (1.4) implies that for $z \in \Sigma$ we have

$$
\tilde{\Psi}_{n+1}\left(z^{*-1}\right)=\left(\boldsymbol{Z}^{*-1}+\boldsymbol{q}_{n}\right) \tilde{\Psi}_{n}\left(z^{*-1}\right) .
$$

Taking the complex conjugate transpose and the inverse simultaneously, we obtain

$$
\tilde{\Psi}_{n+1}\left(z^{*-1}\right)^{\dagger^{-1}}=\frac{\boldsymbol{Z}+\boldsymbol{q}_{n}}{1+\left|q_{n}\right|^{2}} \tilde{\Psi}_{n}\left(z^{*-1}\right)^{\dagger^{-1}} .
$$

Multiplying either side by the positive scalar $w_{n+1}$ satisfying $\left(w_{n+1} / w_{n}\right)=1+\left|q_{n}\right|^{2}$, we arrive at the identity

$$
w_{n+1} \tilde{\Psi}_{n+1}\left(z^{*-1}\right)^{\dagger^{-1}}=\left(\boldsymbol{Z}+\boldsymbol{q}_{n}\right) w_{n} \tilde{\Psi}_{n}\left(z^{*-1}\right)^{\dagger^{-1}},
$$

where, apart from an irrelevant positive constant factor,

$$
w_{n}=\left(1+A^{2}\right)^{n} \prod_{s=n}^{\infty} \frac{1+A^{2}}{1+\left|q_{s}\right|^{2}} .
$$

Observing that the infinite product tends to 1 as $n \rightarrow+\infty$, we obtain the conjugation symmetry property

$$
\tilde{\Psi}_{n}(z)=\left[\left(1+A^{2}\right)^{n} \prod_{s=n}^{\infty} \frac{1+A^{2}}{1+\left|q_{s}\right|^{2}}\right] \tilde{\Psi}_{n}\left(z^{*-1}\right)^{\dagger-1} .
$$

Starting in an analogous way we derive the identity

$$
\hat{w}_{n+1} \tilde{\Phi}_{n+1}\left(z^{*-1}\right)^{\dagger^{-1}}=\left(\boldsymbol{Z}+\boldsymbol{q}_{n}\right) \hat{w}_{n} \tilde{\Phi}_{n}\left(z^{*-1}\right)^{\dagger^{-1}},
$$

where, apart from an irrelevant positive constant factor,

$$
\hat{w}_{n}=\left(1+A^{2}\right)^{n} \prod_{s=-\infty}^{n-1} \frac{1+\left|q_{s}\right|^{2}}{1+A^{2}} .
$$


Observing that the infinite product tends to 1 as $n \rightarrow-\infty$, we obtain the conjugation symmetry property

$$
\tilde{\Phi}_{n}(z)=\left[\left(1+A^{2}\right)^{n} \prod_{s=-\infty}^{n-1} \frac{1+\left|q_{s}\right|^{2}}{1+A^{2}}\right] \tilde{\Phi}_{n}\left(z^{*-1}\right)^{\dagger^{-1}} .
$$

Therefore, for $z \in \mathbb{T}$ the "corrected" fundamental eigensolutions $w_{n}^{-1 / 2} \tilde{\Psi}_{n}(z)$ and $\hat{w}_{n}^{-1 / 2} \tilde{\Phi}_{n}(z)$ belong to the special unitary group $S U(2)$.

Equations (2.9), (3.16), and (3.17) imply that

$$
\begin{aligned}
& \boldsymbol{B}_{l}(z)=\boldsymbol{B}_{l}\left(z^{*-1}\right)^{\dagger^{-1}}\left[\prod_{s=-\infty}^{\infty} \frac{1+\left|q_{s}\right|^{2}}{1+A^{2}}\right], \\
& \boldsymbol{B}_{r}(z)=\boldsymbol{B}_{r}\left(z^{*-1}\right)^{\dagger^{-1}}\left[\prod_{s=-\infty}^{\infty} \frac{1+A^{2}}{1+\left|q_{s}\right|^{2}}\right],
\end{aligned}
$$

where $z \in \Sigma$.

Using (3.10), (3.9d), (3.8b), (3.16), and (3.17) we obtain

$$
\begin{aligned}
& \left(\bar{\psi}_{n}(z) \psi_{n}(z)\right)=\left[1-A^{2} \gamma(z)^{2}\right] \times \\
& \times\left[\left(1+A^{2}\right)^{n} \prod_{s=n}^{\infty} \frac{1+A^{2}}{1+\left|q_{s}\right|^{2}}\right]\left(\bar{\psi}_{n}\left(z^{*-1}\right) \psi_{n}\left(z^{*-1}\right)\right)^{\dagger-1}, \\
& \left(\phi_{n}(z) \bar{\phi}_{n}(z)\right)=\left[1-A^{2} \gamma(z)^{2}\right] \times \\
& \times\left[\left(1+A^{2}\right)^{n} \prod_{s=-\infty}^{n-1} \frac{1+\left|q_{s}\right|^{2}}{1+A^{2}}\right]\left(\phi_{n}\left(z^{*-1}\right) \bar{\phi}_{n}\left(z^{*-1}\right)\right)^{\dagger-1} .
\end{aligned}
$$

With the help of (2.8) and (3.8b) we obtain

$$
\begin{aligned}
\left(\bar{\psi}_{n}(z) \psi_{n}(z)\right) & =\sigma_{2}\left(\bar{\psi}_{n}\left(z^{*-1}\right) \psi_{n}\left(z^{*-1}\right)\right)^{*} \sigma_{2}, \\
\left(\phi_{n}(z) \bar{\phi}_{n}(z)\right) & =\sigma_{2}\left(\phi_{n}\left(z^{*-1}\right) \bar{\phi}_{n}\left(z^{*-1}\right)\right)^{*} \sigma_{2},
\end{aligned}
$$

where the asterisk denotes complex conjugation (without transposition).

d. Jost solutions and analyticity. We now derive the analyticity and continuity properties of the Jost solutions. In (3.10) we need to choose the piecewise $\zeta$-analytic function $\gamma(z)$ in such a way that it has the same $\zeta$-domain of analyticity as the Jost solution.

Theorem 3.1. Assume the hypothesis $\left(\boldsymbol{H}_{0}\right)$ is true and that $q_{n} \neq\left(-1 / q_{r}^{*}\right)$ for every $n \in \mathbb{Z}$. Then the following statements are true:

a. The function $\zeta^{-n} \phi_{n}(z)$ is analytic in $\zeta \in \Lambda^{-}$and continuous in $\zeta \in \overline{\Lambda^{-}}$, except possibly at $\zeta= \pm 1$.

b. The function $\zeta^{n} \bar{\phi}_{n}(z)$ is analytic in $\zeta \in \Lambda^{+}$and continuous in $\zeta \in \overline{\Lambda^{+}}$, except possibly at $\zeta= \pm 1$.

c. The function $\zeta^{-n} \bar{\psi}_{n}(z)$ is analytic in $\zeta \in \Lambda^{+}$and continuous in $\zeta \in \overline{\Lambda^{+}}$, except possibly at $\zeta= \pm 1$.

d. The function $\zeta^{n} \psi_{n}(z)$ is analytic in $\zeta \in \Lambda^{-}$and continuous in $\zeta \in \overline{\Lambda^{-}}$, except possibly at $\zeta= \pm 1$. 
Under the hypothesis $\left(\boldsymbol{H}_{1}\right)$ the continuity can be extended to $\zeta= \pm 1$.

The assumption that $q_{n} \neq\left(-1 / q_{r}^{*}\right)$ for every $n \in \mathbb{Z}$, can only be violated for finitely many $n$. It is only required to prove the analyticity of $\zeta^{-n} \bar{\psi}_{n}(z)$ at $\zeta=0$ and $\zeta^{n} \psi_{n}(\zeta)$ at $\zeta=\infty$. It is also required to derive the first order WKB approximations of $\bar{\psi}_{n}(z)$ near $z=0$ and of $\psi_{n}(z)$ near $z=\infty$.

Proof. Equations (2.5b) and (3.10b) imply that

$$
\begin{aligned}
& \zeta^{-n} \phi_{n}(z)=\left(1+A^{2}\right)^{\frac{n}{2}}\left(\begin{array}{c}
1 \\
\gamma(z) q_{l}^{*}
\end{array}\right)+\zeta^{-1} \sum_{s=-\infty}^{n-1} \boldsymbol{X}_{n, s}^{l}(\zeta) \zeta^{-s} \phi_{s}(z) \\
& \zeta^{n} \bar{\phi}_{n}(z)=\left(1+A^{2}\right)^{\frac{n}{2}}\left(\begin{array}{c}
\gamma(z) q_{l} \\
1
\end{array}\right)+\zeta \sum_{s=-\infty}^{n-1} \overline{\boldsymbol{X}}_{n, s}^{l}(\zeta) \zeta^{s} \bar{\phi}_{s}(z)
\end{aligned}
$$

where

$$
\begin{aligned}
& \left(1+A^{2}\right)^{\frac{s-n+1}{2}} \boldsymbol{X}_{n, s}^{l}(\zeta)=\zeta^{s-n+1} W_{l}(z)\left(\frac{\boldsymbol{Z}+\boldsymbol{q}_{l}}{\sqrt{1+A^{2}}}\right)^{n-s-1} W_{l}(z)^{-1}\left(\boldsymbol{q}_{s}-\boldsymbol{q}_{l}\right) \\
& =\frac{\left(\begin{array}{cc}
\gamma(z)\left[q_{s}^{*}-q_{l}^{*}\right] q_{l}\left[1-\zeta^{2(s-n+1)}\right] & {\left[q_{s}-q_{l}\right]\left[1-A^{2} \gamma(z)^{2} \zeta^{2(s-n+1)}\right]} \\
-\left[q_{s}^{*}-q_{l}^{*}\right]\left[\zeta^{2(s-n+1)}-A^{2} \gamma(z)^{2}\right] & \gamma(z)\left[q_{s}-q_{l}\right] q_{l}^{*}\left[1-\zeta^{2(s-n+1)}\right]
\end{array}\right)}{1-A^{2} \gamma(z)^{2}}, \\
& \left(1+A^{2}\right)^{\frac{s-n+1}{2}} \overline{\boldsymbol{X}}_{n, s}^{l}(\zeta)=\zeta^{n-s-1} W_{l}(z)\left(\frac{\boldsymbol{Z}+\boldsymbol{q}_{l}}{\sqrt{1+A^{2}}}\right)^{n-s-1} W_{l}(z)^{-1}\left(\boldsymbol{q}_{s}-\boldsymbol{q}_{l}\right) \\
& =\frac{\left(\begin{array}{cc}
\gamma(z)\left[q_{s}^{*}-q_{l}^{*}\right] q_{l}\left[\zeta^{2(n-s-1)}-1\right] & {\left[q_{s}-q_{l}\right]\left[\zeta^{2(n-s-1)}-A^{2} \gamma(z)^{2}\right]} \\
-\left[q_{s}^{*}-q_{l}^{*}\right]\left[1-\zeta^{2(n-s-1)} A^{2} \gamma(z)^{2}\right] & \gamma(z)\left[q_{s}-q_{l}\right] q_{l}^{*}\left[\zeta^{2(n-s-1)}-1\right]
\end{array}\right)}{1-A^{2} \gamma(z)^{2}} .
\end{aligned}
$$

The functions $\zeta^{-n} \phi_{n}(z)$ and $\zeta^{n} \bar{\phi}_{n}(z)$ have the analyticity and continuity properties indicated by Theorem 3.1, provided the $\zeta$-domain of analyticity of $\gamma(z)$ matches that of the Jost solution. Finally, we may remove a common factor $\zeta-\zeta^{-1}$ in numerator and denominator [cf. (3.7)] and reduce expressions of the type $\zeta^{2(n-s-1)}-1$ to sums of powers of $\zeta$, at the price of assuming $\left(\boldsymbol{H}_{1}\right)$.

Next, postmultiplying (2.7) by $W_{r}(z)$, and using (3.10a), we get

$$
\begin{gathered}
\zeta^{-n} \bar{\psi}_{n}(z)=\left(1+A^{2}\right)^{\frac{n}{2}}\left(\begin{array}{c}
1 \\
\gamma(z) q_{r}^{*}
\end{array}\right)+\zeta^{-1} \sum_{s=n}^{\infty} \overline{\boldsymbol{X}}_{n, s}^{r}(\zeta) \zeta^{-s} \bar{\psi}_{s}(z), \\
\zeta^{n} \psi_{n}(z)=\left(1+A^{2}\right)^{\frac{n}{2}}\left(\begin{array}{c}
\gamma(z) q_{r} \\
1
\end{array}\right)+\zeta \sum_{s=n}^{\infty} \boldsymbol{X}_{n, s}^{r}(\zeta) \zeta^{s} \psi_{s}(z)
\end{gathered}
$$

where

$$
\begin{aligned}
& \left(1+A^{2}\right)^{\frac{s-n+1}{2}} \overline{\boldsymbol{X}}_{n, s}^{r}(\zeta)=\zeta^{s-n+1} W_{r}(z)\left(\frac{\boldsymbol{Z}+\boldsymbol{q}_{r}}{\sqrt{1+A^{2}}}\right)^{n-s-1} W_{r}(z)^{-1}\left(\boldsymbol{q}_{s}-\boldsymbol{q}_{r}\right)
\end{aligned}
$$

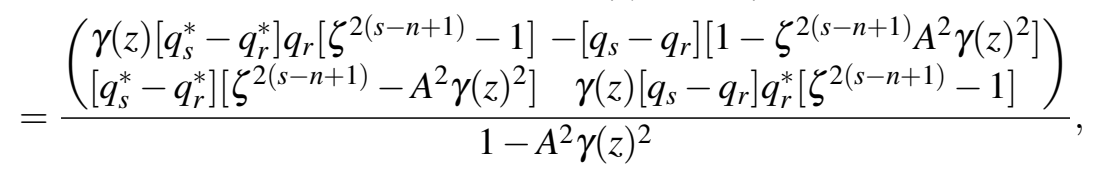




$$
\begin{aligned}
& \left(1+A^{2}\right)^{\frac{s-n+1}{2}} \boldsymbol{X}_{n, s}^{r}(\zeta)=\zeta^{n-s-1} W_{r}(z)\left(\frac{\boldsymbol{Z}+\boldsymbol{q}_{r}}{\sqrt{1+A^{2}}}\right)^{n-s-1} W_{r}(z)^{-1}\left(\boldsymbol{q}_{s}-\boldsymbol{q}_{r}\right)
\end{aligned}
$$

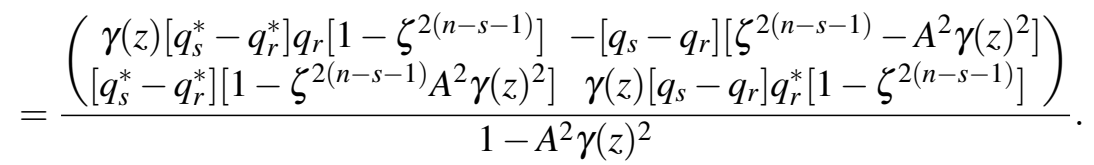

Here the $\zeta$-domain of analyticity of $\gamma(z)$ matches that of the Jost solution.

Seemingly, $\zeta^{-n} \bar{\psi}_{n}(z)$ is not analytic in $\zeta=0$ and $\zeta^{n} \bar{\psi}_{n}(z)$ is not analytic in $\zeta=\infty$, because of the $(1,2)$-entry of $\zeta^{-1} \overline{\boldsymbol{X}}_{n, s}^{r}(\zeta)$ having a pole at $\zeta=0$ and the $(2,1)$-entry of $\zeta \boldsymbol{X}_{n, s}^{r}(\zeta)$ having a pole at $\zeta=\infty$. Writing out the top portion of (3.22a), we get

$$
\begin{aligned}
\zeta^{-n} \bar{\psi}_{n}^{\text {up }}(z) & =\left(1+A^{2}\right)^{\frac{n}{2}} \\
& +\sum_{s=n}^{\infty} \frac{\left(1+A^{2}\right)^{\frac{n-s-1}{2}}}{1-A^{2} \gamma(z)^{2}}\left\{\left[q_{s}^{*}-q_{r}^{*}\right] q_{r} \frac{\gamma(z)}{\zeta}\left[\zeta^{2(s-n+1)}-1\right] \zeta^{-s} \bar{\Psi}_{s}^{\text {up }}(z)\right. \\
& \left.-\left[q_{s}-q_{r}\right]\left[1-\zeta^{2(s-n+1)} A^{2} \gamma(z)^{2}\right] \zeta^{-s-1} \bar{\psi}_{s}^{\text {dn }}(z)\right\},
\end{aligned}
$$

where we observe that $\gamma(z)$ and $\zeta^{-s} \bar{\psi}_{s}^{\text {dn }}(z)$ are odd functions of $\zeta$ and hence can be divided by $\zeta$ without producing a singularity. Using (B.1) and (B.3a) we see that near $\zeta=0$ the coefficient of $\zeta^{-n} \bar{\psi}_{n}^{\text {up }}(z)$ equals $\left(1+A^{2}\right)^{\frac{n}{2}} \frac{1+q_{n}^{*} q_{r}}{1+A^{2}}$ which we assume to be nonzero. The same reasoning applies to the bottom portion of $(3.22 \mathrm{~b})$ at $\zeta=\infty$. Indeed,

$$
\begin{aligned}
\zeta^{-n} \bar{\psi}_{n}^{\mathrm{dn}}(z) & =\left(1+A^{2}\right)^{\frac{n}{2}} \gamma(z) q_{r}^{*} \\
& +\sum_{s=n}^{\infty} \frac{\left(1+A^{2}\right)^{\frac{n-s-1}{2}}}{1-A^{2} \gamma(z)^{2}}\left\{\left[q_{s}^{*}-q_{r}^{*}\right] \frac{\zeta^{2(s-n+1)}-A^{2} \gamma(z)^{2}}{\zeta} \zeta^{-s} \bar{\psi}_{s}^{\text {up }}(z)\right. \\
& \left.+\left[q_{s}-q_{r}\right] q_{r}^{*} \frac{\gamma(z)}{\zeta}\left[\zeta^{2(s-n+1)}-1\right] \zeta^{-s} \bar{\psi}_{s}^{\mathrm{dn}}(z)\right\} .
\end{aligned}
$$

Since the coefficient of $\zeta^{-n} \bar{\psi}_{n}^{\text {dn }}(z)$ near $\zeta=0$ equals $1+O(\zeta)$, we obtain its analyticity near $\zeta=0$. Finally, we may remove a common factor $\zeta-\zeta^{-1}$ in the numerator and denominator [cf. (3.7)] and reduce the expressions of the type $\zeta^{2(n-s-1)}-1$ to sums of powers of $\zeta$, at the price of assuming $\left(\boldsymbol{H}_{1}\right)$.

Let us conclude this section by listing the WKB approximations of the Jost solutions up to first order. This means that we are going to display the approximation of $\zeta^{-n} \phi_{n}(z)$ near $\zeta=0, \zeta^{n} \bar{\phi}_{n}(z)$ near $\zeta=\infty, \zeta^{-n} \bar{\psi}_{n}(z)$ near $\zeta=\infty$, and $\zeta^{n} \psi_{n}(z)$ near $\zeta=0$.

Using (B.2) and (B.4a) we obtain

$$
\zeta^{-n} \phi_{n}(z)=\left(1+A^{2}\right)^{\frac{n}{2}}\left(\begin{array}{l}
1 \\
0
\end{array}\right)-\frac{\left(1+A^{2}\right)^{\frac{n-1}{2}}}{\zeta}\left(\begin{array}{c}
0 \\
q_{n-1}^{*}
\end{array}\right)+O\left(\zeta^{-3}\right) .
$$

Using (B.1) and (B.3a) we obtain instead

$$
\zeta^{n} \bar{\phi}_{n}(z)=\left(1+A^{2}\right)^{\frac{n}{2}}\left(\begin{array}{l}
0 \\
1
\end{array}\right)+\left(1+A^{2}\right)^{\frac{n-1}{2}} \zeta\left(\begin{array}{c}
q_{n-1} \\
0
\end{array}\right)+O\left(\zeta^{3}\right)
$$

where we have exploited the parity symmetry of the Jost solutions. 
The WKB expansions of the other two Jost solutions are more difficult to derive. We confine ourselves to the dominant terms. Let us write

$$
\begin{aligned}
& \zeta^{-n} \bar{\psi}_{n}(z)=\left(1+A^{2}\right)^{\frac{n}{2}}\left(\begin{array}{c}
\bar{C}_{n}^{\text {up }} \\
\bar{C}_{n}^{\text {dn }} \zeta
\end{array}\right)\left[1+O\left(\zeta^{2}\right)\right], \\
& \zeta^{n} \psi_{n}(z)=\left(1+A^{2}\right)^{\frac{n}{2}}\left(\begin{array}{c}
C_{n}^{\text {up }} \zeta^{-1} \\
C_{n}^{\text {dn }}
\end{array}\right)\left[1+O\left(\zeta^{-2}\right)\right],
\end{aligned}
$$

where the constants are as yet unspecified. Put

$$
\begin{aligned}
& \frac{\zeta^{-n} \bar{\psi}_{n}(z)}{\left(1+A^{2}\right)^{\frac{n}{2}}}=\left(\begin{array}{c}
1 \\
\frac{q_{r}^{*}}{\sqrt{1+A^{2}}} \zeta+O\left(\zeta^{3}\right)
\end{array}\right) \\
& +\sum_{s=n}^{\infty} \frac{\left(1+A^{2}\right)^{\frac{-s-1}{2}}}{1+O\left(\zeta^{2}\right)}\left(\begin{array}{cc}
-\left[q_{s}^{*}-q_{r}^{*}\right] \frac{q_{r}}{\sqrt{1+A^{2}}} & -\left[q_{s}-q_{r}\right] \zeta^{-1} \\
{\left[q_{s}^{*}-q_{r}^{*}\right] \zeta\left[\delta_{s, n}-A^{2}\right]\left[q_{s}-q_{r}\right] \frac{-q_{r}^{*}}{\sqrt{1+A^{2}}}}
\end{array}\right)\left(\begin{array}{c}
\bar{C}_{s}^{\text {up }} \\
\bar{C}_{s}^{\text {dn }} \zeta
\end{array}\right), \\
& \frac{\zeta^{n} \psi_{n}(z)}{\left(1+A^{2}\right)^{\frac{n}{2}}}=\left(\begin{array}{c}
\frac{q_{r} \zeta^{-1}}{\sqrt{1+A^{2}}}+O\left(\zeta^{-3}\right) \\
1
\end{array}\right) \\
& +\sum_{s=n}^{\infty} \frac{\left(1+A^{2}\right)^{\frac{-s-1}{2}}}{1+O\left(\zeta^{-2}\right)}\left(\begin{array}{cc}
{\left[q_{s}^{*}-q_{r}^{*}\right] \frac{-q_{r}}{\sqrt{1+A^{2}}}} & {\left[q_{s}-q_{r}\right]\left(A^{2}-\delta_{s, n}\right) \zeta^{-1}} \\
{\left[q_{s}^{*}-q_{r}^{*}\right] \zeta} & {\left[q_{s}-q_{r}\right] \frac{-q_{r}^{*}}{\sqrt{1+A^{2}}}}
\end{array}\right)\left(\begin{array}{c}
C_{s}^{\mathrm{up}} \zeta^{-1} \\
C_{s}^{\mathrm{dn}}
\end{array}\right) .
\end{aligned}
$$

We then arrive at the following two biinfinite upper triangular systems:

$$
\begin{aligned}
& \frac{1+q_{n}^{*} q_{r}}{1+A^{2}} \bar{C}_{n}^{\mathrm{up}}=1 \\
& +\sum_{s=n+1}^{\infty}\left(1+A^{2}\right)^{\frac{-s-1}{2}}\left\{\frac{-\left[q_{s}^{*}-q_{r}^{*}\right] q_{r}}{\sqrt{1+A^{2}}} \bar{C}_{s}^{\mathrm{up}}-\left[q_{s}-q_{r}\right] \bar{C}_{s}^{\mathrm{dn}}\right\}, \\
& \frac{1+q_{n} q_{r}^{*}}{1+A^{2}} \bar{C}_{n}^{\mathrm{dn}}=\frac{q_{r}^{*}}{\sqrt{1+A^{2}}} \\
& +\sum_{s=n+1}^{\infty}\left(1+A^{2}\right)^{\frac{-s-1}{2}}\left\{-\left[q_{s}^{*}-q_{r}^{*}\right] A^{2} \bar{C}_{s}^{\mathrm{up}}-\frac{\left[q_{s}-q_{r}\right] q_{r}^{*}}{\sqrt{1+A^{2}}} \bar{C}_{s}^{\mathrm{dn}}\right\}, \\
& \frac{1+q_{n}^{*} q_{r}}{1+A^{2}} C_{n}^{\mathrm{up}}=\frac{q_{r}}{\sqrt{1+A^{2}}} \\
& +\sum_{s=n+1}^{\infty}\left(1+A^{2}\right)^{\frac{-s-1}{2}}\left\{\frac{-\left[q_{s}^{*}-q_{r}^{*}\right] q_{r}}{\sqrt{1+A^{2}}} C_{s}^{\mathrm{up}}+\left[q_{s}-q_{r}\right] A^{2} C_{s}^{\mathrm{dn}}\right\}, \\
& \frac{1+q_{n} q_{r}^{*}}{1+A^{2}} C_{n}^{\mathrm{dn}}=1 \\
& +\sum_{s=n+1}^{\infty}\left(1+A^{2}\right)^{\frac{-s-1}{2}}\left\{\left[q_{s}^{*}-q_{r}^{*}\right] C_{s}^{\mathrm{up}}-\frac{\left[q_{s}-q_{r}\right] q_{r}^{*}}{\sqrt{1+A^{2}}} C_{s}^{\mathrm{dn}}\right\} .
\end{aligned}
$$

By contraction mapping, (3.25) are easily seen to be uniquely solvable in $\ell^{p}(1 \leq p \leq+\infty)$ for large enough $n$. Their unique solvability can then be extended to smaller $n$ one step at the time, provided $q_{n} \neq\left[-1 / q_{r}^{*}\right]$ for $n \in \mathbb{Z}$. 


\section{Scattering coefficients}

In this section we study the scattering coefficients. Using (2.9), (3.5), and (3.10) we obtain for $\zeta \in \mathbb{T}$

$$
\begin{aligned}
\left(\phi_{n}(z) \bar{\phi}_{n}(z)\right) & =\left(\bar{\psi}_{n}(z) \psi_{n}(z)\right) S(z), \\
\left(\bar{\psi}_{n}(z) \psi_{n}(z)\right) & =\left(\phi_{n}(z) \bar{\phi}_{n}(z)\right) \bar{S}(z),
\end{aligned}
$$

where

$$
\begin{aligned}
& S(z)=\left(\begin{array}{ll}
a(z) & \bar{b}(z) \\
b(z) & \bar{a}(z)
\end{array}\right)=W_{r}(z)^{-1} \boldsymbol{B}_{l}(z) W_{l}(z), \\
& \bar{S}(z)=\left(\begin{array}{ll}
\bar{c}(z) & d(z) \\
\bar{d}(z) & c(z)
\end{array}\right)=W_{l}(z)^{-1} \boldsymbol{B}_{r}(z) W_{r}(z) .
\end{aligned}
$$

Then for $\zeta \in \mathbb{T}$ we get

$$
\begin{aligned}
& \operatorname{det} S(z)=\frac{\operatorname{det} W_{l}(z)}{\operatorname{det} W_{r}(z)} \operatorname{det} \boldsymbol{B}_{l}(z)=\prod_{s=-\infty}^{\infty} \frac{1+\left|q_{s}\right|^{2}}{1+A^{2}}, \\
& \operatorname{det} \bar{S}(z)=\frac{\operatorname{det} W_{r}(z)}{\operatorname{det} W_{l}(z)} \operatorname{det} \boldsymbol{B}_{r}(z)=\prod_{s=-\infty}^{\infty} \frac{1+A^{2}}{1+\left|q_{s}\right|^{2}} .
\end{aligned}
$$

Equations (4.1) and (3.15) imply the parity symmetry relations

$$
\sigma_{3} S(-z) \sigma_{3}=S(z), \quad \sigma_{3} \bar{S}(-z) \sigma_{3}=\bar{S}(z)
$$

so that the diagonal entries $a(z), \bar{a}(z), c(z)$, and $\bar{c}(z)$ are even functions of $z$ and $\zeta$ and the offdiagonal entries $b(z), \bar{b}(z), d(z)$, and $\bar{d}(z)$ are odd functions of $z$ and $\zeta$. Next, using (3.9d), (3.18), and (4.2) we get

$$
\begin{aligned}
& S\left(z^{*-1}\right)^{\dagger^{-1}}=S(z) \prod_{s=-\infty}^{\infty} \frac{1+A^{2}}{1+\left|q_{s}\right|^{2}}=s S(z), \\
& \bar{S}\left(z^{*-1}\right)^{\dagger^{-1}}=\bar{S}(z) \prod_{s=-\infty}^{\infty} \frac{1+\left|q_{s}\right|^{2}}{1+A^{2}}=\frac{\bar{S}(z)}{s},
\end{aligned}
$$

where $s=\prod_{s=-\infty}^{\infty} \frac{1+A^{2}}{1+\left|q_{s}\right|^{2}}$. Thus for $z \in \mathbb{T}$ the matrices $s^{1 / 2} S(z)$ and $s^{-1 / 2} \bar{S}(z)$ belong to $S U(2)$. Finally, using (3.8b), (4.3), and (4.5) we easily obtain

$$
\begin{aligned}
& \left(\begin{array}{ll}
a(z) & \bar{b}(z) \\
b(z) & \bar{a}(z)
\end{array}\right)=\left(\begin{array}{cc}
\bar{a}\left(z^{*-1}\right)^{*} & -b\left(z^{*-1}\right)^{*} \\
-\bar{b}\left(z^{*-1}\right)^{*} & a\left(z^{*-1}\right)^{*}
\end{array}\right), \\
& \left(\begin{array}{cc}
\bar{c}(z) & d(z) \\
\bar{d}(z) & c(z)
\end{array}\right)=\left(\begin{array}{cc}
c\left(z^{*-1}\right)^{*} & -\bar{d}\left(z^{*-1}\right)^{*} \\
-d\left(z^{*-1}\right)^{*} & \bar{c}\left(z^{*-1}\right)^{*}
\end{array}\right) .
\end{aligned}
$$


a. Wronskian relations. Using (4.1), (4.2), and Proposition 2.1 we derive the following determinant relations:

$$
\begin{aligned}
a(z) & =\frac{\operatorname{det}\left(\phi_{n}(z) \psi_{n}(z)\right)}{\operatorname{det}\left(\bar{\psi}_{n}(z) \psi_{n}(z)\right)} \\
& =\frac{\prod_{s=n}^{\infty}\left[\left(1+\left|q_{s}\right|^{2}\right) /\left(1+A^{2}\right)\right]}{\left(1+A^{2}\right)^{n}\left[1-A^{2} \gamma(z)^{2}\right]} \operatorname{det}\left(\phi_{n}(z) \psi_{n}(z)\right), \\
\bar{a}(z) & =\frac{\operatorname{det}\left(\bar{\psi}_{n}(z) \bar{\phi}_{n}(z)\right)}{\operatorname{det}\left(\bar{\psi}_{n}(z) \psi_{n}(z)\right)} \\
& =\frac{\prod_{s=n}^{\infty}\left[\left(1+\left|q_{s}\right|^{2}\right) /\left(1+A^{2}\right)\right]}{\left(1+A^{2}\right)^{n}\left[1-A^{2} \gamma(z)^{2}\right]} \operatorname{det}\left(\bar{\psi}_{n}(z) \bar{\phi}_{n}(z)\right), \\
b(z) & =\frac{\operatorname{det}\left(\bar{\psi}_{n}(z) \phi_{n}(z)\right)}{\operatorname{det}\left(\bar{\psi}_{n}(z) \psi_{n}(z)\right)} \\
& =\frac{\prod_{s=n}^{\infty}\left[\left(1+\left|q_{s}\right|^{2}\right) /\left(1+A^{2}\right)\right]}{\left(1+A^{2}\right)^{n}\left[1-A^{2} \gamma(z)^{2}\right]} \operatorname{det}\left(\bar{\psi}_{n}(z) \phi_{n}(z)\right), \\
\bar{b}(z) & =\frac{\operatorname{det}\left(\bar{\phi}_{n}(z) \psi_{n}(z)\right)}{\operatorname{det}\left(\bar{\psi}_{n}(z) \psi_{n}(z)\right)} \\
& =\frac{\prod_{s=n}^{\infty}\left[\left(1+\left|q_{s}\right|^{2}\right) /\left(1+A^{2}\right)\right]}{\left(1+A^{2}\right)^{n}\left[1-A^{2} \gamma(z)^{2}\right]} \operatorname{det}\left(\bar{\phi}_{n}(z) \psi_{n}(z)\right) .
\end{aligned}
$$

In the same way,

$$
\begin{aligned}
& c(z)=\frac{\operatorname{det}\left(\phi_{n}(z) \psi_{n}(z)\right)}{\operatorname{det}\left(\phi_{n}(z) \bar{\phi}_{n}(z)\right)}=\frac{\operatorname{det}\left(\phi_{n}(z) \psi_{n}(z)\right)}{\left[1-A^{2} \gamma(z)^{2}\right]\left(1+A^{2}\right)^{n} \prod_{s=-\infty}^{n-1} \frac{1+\left|q_{s}\right|^{2}}{1+A^{2}}}, \\
& \bar{c}(z)=\frac{\operatorname{det}\left(\bar{\phi}_{n}(z) \bar{\psi}_{n}(z)\right)}{\operatorname{det}\left(\phi_{n}(z) \bar{\phi}_{n}(z)\right)}=\frac{\operatorname{det}\left(\bar{\phi}_{n}(z) \bar{\psi}_{n}(z)\right)}{\left[1-A^{2} \gamma(z)^{2}\right]\left(1+A^{2}\right)^{n} \prod_{s=-\infty}^{n-1} \frac{1+\left|q_{s}\right|^{2}}{1+A^{2}}}, \\
& d(z)=\frac{\operatorname{det}\left(\bar{\phi}_{n}(z) \psi_{n}(z)\right)}{\operatorname{det}\left(\phi_{n}(z) \bar{\phi}_{n}(z)\right)}=\frac{\operatorname{det}\left(\bar{\phi}_{n}(z) \psi_{n}(z)\right)}{\left[1-A^{2} \gamma(z)^{2}\right]\left(1+A^{2}\right)^{n} \prod_{s=-\infty}^{n-1} \frac{1+\left|q_{s}\right|^{2}}{1+A^{2}}}, \\
& \bar{d}(z)=\frac{\operatorname{det}\left(\phi_{n}(z) \bar{\psi}_{n}(z)\right)}{\operatorname{det}\left(\phi_{n}(z) \bar{\phi}_{n}(z)\right)}=\frac{\operatorname{det}\left(\phi_{n}(z) \bar{\psi}_{n}(z)\right)}{\left[1-A^{2} \gamma(z)^{2}\right]\left(1+A^{2}\right)^{n} \prod_{s=-\infty}^{n-1} \frac{1+\left|q_{s}\right|^{2}}{1+A^{2}}} .
\end{aligned}
$$

Therefore, by the analyticity of two Jost solutions on a common domain $\mathbb{K}^{ \pm}, a(z)$ and $c(z)$ are analytic in $z \in \mathbb{K}^{-}$, whereas $\bar{a}(z)$ and $\bar{c}(z)$ are analytic in $z \in \mathbb{K}^{-}$.

Equations (4.7) imply that

$$
\begin{aligned}
& \frac{a(z)}{c(z)}=\frac{\operatorname{det}\left(\phi_{n}(z) \bar{\phi}_{n}(z)\right)}{\operatorname{det}\left(\bar{\psi}_{n}(z) \psi_{n}(z)\right)}=\prod_{s=-\infty}^{\infty} \frac{1+\left|q_{s}\right|^{2}}{1+A^{2}}, \\
& \frac{\bar{a}(z)}{\bar{c}(z)}=\frac{\operatorname{det}\left(\phi_{n}(z) \bar{\phi}_{n}(z)\right)}{\operatorname{det}\left(\bar{\psi}_{n}(z) \psi_{n}(z)\right)}=\prod_{s=-\infty}^{\infty} \frac{1+\left|q_{s}\right|^{2}}{1+A^{2}} .
\end{aligned}
$$

Thus $a(z)$ and $c(z)$ have the same zeros (with the same zero order) for $z \in \mathbb{K}^{+}$, while $\bar{a}(z)$ and $\bar{c}(z)$ have the same zeros (with the same zero order) for $z \in \mathbb{K}^{-}$. 
When multiplying each of the quantities $a(z), \bar{a}(z), b(z), \bar{b}(z), c(z), \bar{c}(z), d(z)$, and $\bar{d}(z)$ by $1-A^{2} \gamma(z)^{2}$, we have a finite limit as $z$ approaches one of the four points $\pm \sqrt{1+A^{2}} \pm A$ from within their domains.

Using (3.5), (4.1), and (4.2) we easily deduce that

$$
\begin{aligned}
& {\left[1-A^{2} \gamma(z)^{2}\right] a(z) \sim\left(1+A^{2}\right)^{-\frac{n}{2}}\left\{\zeta^{-n} \phi_{n}^{\text {up }}(z)-\gamma(z) q_{r} \zeta^{-n} \phi_{n}^{\text {dn }}(z)\right\},} \\
& {\left[1-A^{2} \gamma(z)^{2}\right] \bar{a}(z) \sim\left(1+A^{2}\right)^{-\frac{n}{2}}\left\{\zeta^{n} \bar{\phi}_{n}^{\mathrm{dn}}(z)-\gamma(z) q_{r}^{*} \zeta^{n} \bar{\phi}_{n}^{\text {up }}(z)\right\},} \\
& {\left[1-A^{2} \gamma(z)^{2}\right] c(z) \sim\left(1+A^{2}\right)^{-\frac{n}{2}}\left\{\zeta^{n} \psi_{n}^{\mathrm{dn}}(z)-\gamma(z) q_{l}^{*} \zeta^{n} \psi_{n}^{\text {up }}(z)\right\},} \\
& {\left[1-A^{2} \gamma(z)^{2}\right] \bar{c}(z) \sim\left(1+A^{2}\right)^{-\frac{n}{2}}\left\{\zeta^{-n} \bar{\psi}_{n}^{\text {up }}(z)-\gamma(z) q_{l} \zeta^{-n} \bar{\psi}_{n}^{\mathrm{dn}}(z)\right\},}
\end{aligned}
$$

as well as

$$
\begin{aligned}
{\left[1-A^{2} \gamma(z)^{2}\right] \zeta^{-2 n} b(z) } & \sim\left(1+A^{2}\right)^{-\frac{n}{2}}\left\{\zeta^{-n} \phi_{n}^{\mathrm{dn}}(z)-\gamma(z) q_{r}^{*} \zeta^{-n} \phi_{n}^{\text {up }}(z)\right\}, \\
{\left[1-A^{2} \gamma(z)^{2}\right] \zeta^{2 n} \bar{b}(z) } & \sim\left(1+A^{2}\right)^{-\frac{n}{2}}\left\{\zeta^{n} \bar{\phi}_{n}^{\text {up }}(z)-\gamma(z) q_{r} \zeta^{n} \bar{\phi}_{n}^{\mathrm{dn}}(z)\right\} \\
{\left[1-A^{2} \gamma(z)^{2}\right] \zeta^{2 n} d(z) } & \sim\left(1+A^{2}\right)^{-\frac{n}{2}}\left\{\zeta^{n} \psi_{n}^{\mathrm{up}}(z)-\gamma(z) q_{l} \zeta^{n} \psi_{n}^{\mathrm{dn}}(z)\right\}, \\
{\left[1-A^{2} \gamma(z)^{2}\right] \zeta^{-2 n} \bar{d}(z) } & \sim\left(1+A^{2}\right)^{-\frac{n}{2}}\left\{\zeta^{-n} \bar{\psi}_{n}^{\mathrm{dn}}(z)-\gamma(z) q_{l}^{*} \zeta^{-n} \bar{\psi}_{n}^{\mathrm{up}}(z)\right\}
\end{aligned}
$$

Here $n \rightarrow-\infty$ in (4.9a), (4.9b), (4.9e), and (4.9f) and $n \rightarrow+\infty$ in the other four equations. Because of (3.23) and (3.24), $a(z) \rightarrow 1$ and $c(z) \rightarrow C_{n}^{\mathrm{dn}}$ as $z \rightarrow \infty$ and $\bar{a}(z) \rightarrow 1$ and $\bar{c}(z) \rightarrow \bar{C}_{n}^{\mathrm{up}}$ as $z \rightarrow 0$. With the help of (4.8) we prove that $c(z)$ as $z \rightarrow \infty$ and $\bar{c}(z)$ as $z \rightarrow 0$ both converge to the positive number $\prod_{s=-\infty}^{\infty}\left[\left(1+A^{2}\right) /\left(1+\left|q_{s}\right|^{2}\right)\right]$. Consequently,

$$
C_{n}^{\mathrm{dn}}=\bar{C}_{n}^{\mathrm{up}}=\prod_{s=-\infty}^{\infty} \frac{1+A^{2}}{1+\left|q_{s}\right|^{2}} .
$$

b. Reflection and transmission coefficients. For future reference we define the reflection coefficients from the right by

$$
\begin{array}{lll}
\rho(z)=\frac{b(z)}{a(z)}, & \rho^{\mathrm{up}}(s)=\frac{b\left(s+i 0^{+}\right)}{a\left(s+i 0^{+}\right)}, & \rho^{\mathrm{dn}}(s)=\frac{b\left(s-i 0^{+}\right)}{a\left(s-i 0^{+}\right)}, \\
\bar{\rho}(z)=\frac{\bar{b}(z)}{\bar{a}(z)}, & \bar{\rho}^{\mathrm{up}}(s)=\frac{\bar{b}\left(s+i 0^{+}\right)}{\bar{a}\left(s+i 0^{+}\right)}, & \bar{\rho}^{\mathrm{dn}}(s)=\frac{\bar{b}\left(s-i 0^{+}\right)}{\bar{a}\left(s-i 0^{+}\right)},
\end{array}
$$

and the reflection coefficients from the left by

$$
\begin{aligned}
& r(z)=\frac{d(z)}{c(z)}=-\frac{\bar{b}(z)}{a(z)}, \quad r^{\mathrm{u} / \mathrm{dn}}(s)=\frac{d\left(s \pm i 0^{+}\right)}{c\left(s \pm i 0^{+}\right)}=-\frac{\bar{b}\left(s \pm i 0^{+}\right)}{a\left(s \pm i 0^{+}\right)}, \\
& \bar{r}(z)=\frac{\bar{d}(z)}{\bar{c}(z)}=-\frac{b(z)}{\bar{a}(z)}, \quad \bar{r}^{\mathrm{u} / \mathrm{dn}}(s)=\frac{\bar{d}\left(s \pm i 0^{+}\right)}{\bar{c}\left(s \pm i 0^{+}\right)}=-\frac{b\left(s \pm i 0^{+}\right)}{\bar{a}\left(s \pm i 0^{+}\right)},
\end{aligned}
$$

where $z \in \mathbb{T}$ and $\left|s \pm \sqrt{1+A^{2}}\right|<A$. Note that in order to express $r(z)$ and $\bar{r}(z)$ in terms of the entries of $S(z)$, we have used that $S(z) \bar{S}(z)=I_{2}$. The coefficients $1 / a(z), 1 / \bar{a}(z), 1 / a\left(s \pm i 0^{+}\right)$, and $1 / \bar{a}\left(s \pm i 0^{+}\right)$are usually referred to as (right) transmission coefficients. Similarly. $1 / c(z), 1 / \bar{c}(z)$, $1 / c\left(s \pm i 0^{+}\right)$, and $1 / \bar{c}\left(s \pm i 0^{+}\right)$are called (left) transmission coefficients. Clearly, the reflection 
coefficients are odd functions of $z$ and $\zeta$ and the transmission coefficients even functions of $z$ and $\zeta$. Moreover,

$$
\rho\left(z^{*-1}\right)^{*}=-\bar{\rho}(z), \quad r\left(z^{*-1}\right)^{*}=-\bar{r}(z) .
$$

c. Discrete eigenvalues. A discrete eigenvalue is a value of $z \in \mathbb{K}^{+} \cup \mathbb{K}^{-}$(corresponding to $\zeta \in \Lambda^{+} \cup \Lambda^{-}$) for which there exists a nontrivial solution $\left\{v_{n}\right\}_{n=-\infty}^{\infty}$ to (1.4) such that $\sum_{n=-\infty}^{\infty}\left\|v_{n}\right\|^{2}$ converges. These solutions occur for $z \in \mathbb{K}^{+}$iff $\phi_{n}(z)$ and $\psi_{n}(z)$ are linearly dependent (i.e., iff $a(z)=0$ ). These solutions occur for $z \in \mathbb{K}^{-}$iff $\bar{\psi}_{n}(z)$ and $\bar{\phi}_{n}(z)$ are linearly dependent (i.e., $\bar{a}(z)=$ $0)$. Equations (3.10) together with the linear dependence imply that the eigenfunctions $\left\{v_{n}\right\}_{n=-\infty}^{\infty}$ are exponentially decaying as $n \rightarrow \pm \infty$. Moreover, the eigenvalues have finite algebraic multiplicity, are at most countably infinite in number, and their $\zeta$-images can only accumulate at points of the unit $\zeta$ circle, but never at zero or at infinity. Here we observe that $a(\infty), c(\infty), \bar{a}(0)$, and $\bar{c}(0)$ are nonzero. Parity symmetry ensures that the discrete eigenvalues occur in \pm pairs. Moreover, the conjugation symmetry $z \mapsto z^{*-1}$ does not alter the set of discrete eigenvalues [cf. (4.5)]. In other words, the discrete eigenvalues occur in quadruplets $\left\{ \pm z, \pm z^{*-1}\right\}$ with $z \notin \Sigma$, all four of them having the same Jordan structure.

The algebraic multiplicity of each discrete eigenvalue $z$ coincides with the multiplicity of $z \in \mathbb{K}^{+}$ as a zero of $a(z)$ or the multiplicity of $z \in \mathbb{K}^{-}$as a zero of $\bar{a}(z)$. In Section 6 we shall assume that the discrete eigenvalues are all algebraically simple. Under this assumption, for any such zero $z_{k} \in \mathbb{K}^{-}$ there exists a complex nonzero proportionality constant $b_{k}$ between the Jost solutions $\phi_{n}\left(z_{k}\right)$ and $\psi_{n}\left(z_{k}\right): \phi_{n}\left(z_{k}\right)=b_{k} \psi_{n}\left(z_{k}\right)$. Denoting the residue of $1 / a(z)$ at $\zeta=\zeta\left(z_{k}\right)$ by $\tau_{k}$ and introducing the norming constant $C_{k}=\tau_{k} b_{k}$, we obtain

$$
\psi_{n}\left(z_{k}\right) C_{k}=\lim _{z \rightarrow z_{k}}\left(\zeta-\zeta\left(z_{k}\right)\right) \frac{\phi_{n}(z)}{a(z)}=\phi_{n}\left(z_{k}\right) \tau_{k} .
$$

Similarly, for any such zero $\bar{z}_{k} \in \mathbb{K}^{+}$there exists a complex nonzero proportionality constant $\bar{b}_{k}$ between the Jost solutions $\bar{\psi}_{n}\left(\bar{z}_{k}\right)$ and $\bar{\phi}_{n}\left(\bar{z}_{k}\right): \bar{\phi}_{n}\left(\bar{z}_{k}\right)=\bar{b}_{k} \bar{\psi}_{n}\left(\bar{z}_{k}\right)$. Denoting the residue of $1 / \bar{a}(z)$ at $\zeta=\zeta\left(\bar{z}_{k}\right)$ by $\bar{\tau}_{k}$ and introducing the norming constant $\bar{C}_{k}$ by $\bar{C}_{k}=\bar{\tau}_{k} \bar{b}_{k}$, we obtain

$$
\bar{\psi}_{n}\left(\bar{z}_{k}\right) \bar{C}_{k}=\lim _{z \rightarrow \bar{z}_{k}}\left(\zeta-\zeta\left(\bar{z}_{k}\right)\right) \frac{\bar{\phi}_{n}(\bar{z})}{\bar{a}(z)}=\bar{\phi}_{n}\left(\bar{z}_{k}\right) \bar{\tau}_{k}
$$

In the same way we define the norming constants

$$
\begin{aligned}
& \phi_{n}\left(z_{k}\right) D_{k}=\lim _{z \rightarrow z_{k}}\left(\zeta-\zeta\left(z_{k}\right)\right) \frac{\psi_{n}(z)}{c(z)}=\psi_{n}\left(z_{k}\right) \hat{\tau}_{k}, \\
& \bar{\phi}_{n}\left(\bar{z}_{k}\right) \bar{D}_{k}=\lim _{z \rightarrow \bar{z}_{k}}\left(\zeta-\zeta\left(\bar{z}_{k}\right)\right) \frac{\bar{\psi}_{n}(\bar{z})}{\bar{c}(z)}=\bar{\psi}_{n}\left(\bar{z}_{k}\right) \hat{\bar{\tau}}_{k},
\end{aligned}
$$

where $D_{k}=\left[\hat{\tau}_{k} / b_{k}\right], \bar{D}_{k}=\left[\hat{\bar{\tau}}_{k} / \bar{b}_{k}\right]$, and $\hat{\tau}_{k}$ and $\hat{\bar{\tau}}_{k}$ are the residues of $1 / c(z)$ and $1 / \bar{c}(z)$ at $\zeta=\zeta\left(z_{k}\right)$ and $\zeta=\zeta\left(\bar{z}_{k}\right)$, respectively.

To study the impact of parity symmetry on the norming constants, we order the discrete eigenvalues in such a way that

$$
z_{-k}=-z_{k}, \quad \bar{z}_{-k}=-\bar{z}_{k},
$$


skipping the subscript $k=0$. Since the transmission coefficients are even functions of $\zeta$, we have

$$
\tau_{-k}=-\tau_{k}, \quad \bar{\tau}_{-k}=-\bar{\tau}_{k}, \quad \hat{\tau}_{-k}=-\hat{\tau}_{k}, \quad \hat{\bar{\tau}}_{-k}=-\hat{\bar{\tau}}_{k}
$$

Writing (4.14a) with $k$ replaced by $-k$ in the form

$$
\left(\begin{array}{c}
-\psi_{n}^{\text {up }}\left(z_{k}\right) \\
\psi_{n}^{\mathrm{dn}}\left(z_{k}\right)
\end{array}\right) C_{-k}=\left(\begin{array}{c}
\phi_{n}^{\text {up }}\left(z_{k}\right) \\
-\phi_{n}^{\operatorname{dn}}\left(z_{k}\right)
\end{array}\right)\left(-\tau_{k}\right)
$$

and doing the same thing for (4.14b)-(4.14d), we obtain

$$
C_{-k}=C_{k}, \quad \bar{C}_{-k}=\bar{C}_{k}, \quad D_{-k}=D_{k}, \quad \bar{D}_{-k}=\bar{D}_{k} .
$$

Next, to study the effect of circle conjugation symmetry on the norming constants, we relate the ordering of the eigenvalues $z_{k}$ and $\bar{z}_{k}$ as follows: $\bar{z}_{k}=\left[z_{k}\right]^{{ }^{-1}}$. With the help of (4.6) we then get

$$
\bar{\tau}_{k}=-\frac{\tau_{k}^{*}}{\zeta\left(z_{k}\right)^{2}}=-\zeta\left(\bar{z}_{k}\right)^{2} \tau_{k}^{*}, \quad \hat{\bar{\tau}}_{k}=-\frac{\hat{\tau}_{k}^{*}}{\zeta\left(z_{k}\right)^{2}}=-\zeta\left(\bar{z}_{k}\right)^{2} \hat{\tau}_{k}^{*}
$$

Using (3.20) we may write (4.14a) in the form

$$
\left(\begin{array}{c}
-\overline{\boldsymbol{\Psi}}_{n}^{\mathrm{dn}}\left(\bar{z}_{k}\right)^{*} \\
\overline{\boldsymbol{\psi}}_{n}^{\mathrm{up}}\left(\bar{z}_{k}\right)^{*}
\end{array}\right) C_{k}=\left(\begin{array}{c}
-\bar{\phi}_{n}^{\mathrm{dn}}\left(\bar{z}_{k}\right)^{*} \\
\bar{\phi}_{n}^{\mathrm{un}}\left(\bar{z}_{k}\right)^{*}
\end{array}\right) \tau_{k}
$$

which coincides with (4.14b) with $\bar{C}_{k}$ and $\bar{\tau}_{k}$ replaced by $C_{k}^{*}$ and $\tau_{k}^{*}$, respectively. Premultiplying by $i \sigma_{2}$ and taking complex conjugates while using (4.16) and (4.14b), we obtain $\bar{C}_{k}=\left(\left[C_{k}\right]^{*} / \tau_{k}^{* 2}\right)$. Doing the same thing to (4.14c), we finally obtain with the help of (4.16) and (4.14d)

$$
\bar{C}_{k}=\frac{\left[C_{k}\right]^{*}}{\zeta\left(z_{k}\right)^{* 2}}=\zeta\left(\bar{z}_{k}\right)^{2}\left[C_{k}\right]^{*}, \quad \bar{D}_{k}=\frac{\left[D_{k}\right]^{*}}{\zeta\left(z_{k}\right)^{* 2}}=\zeta\left(\bar{z}_{k}\right)^{2}\left[D_{k}\right]^{*}
$$

d. Spectral singularities. A spectral singularity is a value of $z \in \mathbb{T}$ for which $a(z)=0$ [or, equivalently, $\bar{a}(z)=0$ ], or a value of $z=s \pm i 0^{+} \in \Sigma \backslash \mathbb{T}$ for which $a^{ \pm}(s)=0$ [or, equivalently, $\bar{a}^{ \pm}(s)=0$ ]. Under the assumption of absence of spectral singularities, the reflection and transmission coefficients are continuous in $\zeta \in \mathbb{T}$.

\section{Triangular representations}

We seek the following triangular representations:

$$
\begin{aligned}
& \tilde{\Psi}_{n}(z)=\sum_{s=n}^{\infty}\left(1+A^{2}\right)^{\frac{n-s}{2}} \boldsymbol{K}(n, s)\left(\boldsymbol{Z}+\boldsymbol{q}_{r}\right)^{s}, \\
& \tilde{\Phi}_{n}(z)=\left(\boldsymbol{Z}+\boldsymbol{q}_{l}\right)^{n}+\sum_{s=-\infty}^{n-1}\left(1+A^{2}\right)^{\frac{n-s}{2}} \boldsymbol{M}(n, s)\left(\boldsymbol{Z}+\boldsymbol{q}_{l}\right)^{s} .
\end{aligned}
$$


where $\boldsymbol{K}(n, n)$ need not be the identity matrix. Putting $\boldsymbol{M}(n, n)=I_{2}$ we can write (5.1b) in a form similar to (5.1a). The representations (5.1) can also be written as follows:

$$
\begin{gathered}
\frac{\overline{\boldsymbol{\Psi}}_{n}(z)}{\left(1+A^{2}\right)^{n / 2}}=\sum_{s=n}^{\infty} \zeta^{s} \boldsymbol{K}(n, s)\left(\begin{array}{c}
1 \\
\gamma(z) q_{r}^{*}
\end{array}\right), \\
\frac{\psi_{n}(z)}{\left(1+A^{2}\right)^{n / 2}}=\sum_{s=n}^{\infty} \zeta^{-s} \boldsymbol{K}(n, s)\left(\begin{array}{c}
\gamma(z) q_{r} \\
1
\end{array}\right), \\
\frac{\phi_{n}(z)}{\left(1+A^{2}\right)^{n / 2}}=\sum_{s=-\infty}^{n} \zeta^{s} \boldsymbol{M}(n, s)\left(\begin{array}{c}
1 \\
\gamma(z) q_{l}^{*}
\end{array}\right), \\
\frac{\bar{\phi}_{n}(z)}{\left(1+A^{2}\right)^{n / 2}}=\sum_{s=-\infty}^{n} \zeta^{-s} \boldsymbol{M}(n, s)\left(\begin{array}{c}
\gamma(z) q_{l} \\
1
\end{array}\right),
\end{gathered}
$$

where $\boldsymbol{M}(n, n)=I_{2}$. By (3.24), $\boldsymbol{K}(n, n)$ is the diagonal matrix satisfying

$$
\boldsymbol{K}(n, n)=\left(\begin{array}{cc}
\bar{C}_{n}^{\mathrm{up}} & 0 \\
0 & C_{n}^{\mathrm{dn}}
\end{array}\right)=\left[\prod_{s=-\infty}^{\infty} \frac{1+A^{2}}{1+\left|q_{s}\right|^{2}}\right] I_{2} .
$$

Theorem 5.1. Assume the hypothesis $\left(\boldsymbol{H}_{1}\right)$ is true and that $q_{n} \neq\left(-1 / q_{r}^{*}\right)$ for every $n \in \mathbb{Z}$. Then the fundamental eigensolutions $\tilde{\Psi}_{n}(z)$ and $\tilde{\Phi}_{n}(z)$ allow the triangular representations $(5.1)$, where the series

$$
\sum_{s=n+1}^{\infty}\|\boldsymbol{K}(n, s)\|^{2}+\sum_{s=-\infty}^{n-1}\|\boldsymbol{M}(n, s)\|^{2}
$$

converges and the parity symmetry relations

$$
\boldsymbol{K}(n, s)=(-1)^{n-s} \sigma_{3} \boldsymbol{K}(n, s) \sigma_{3}, \quad \boldsymbol{M}(n, s)=(-1)^{n-s} \sigma_{3} \boldsymbol{M}(n, s) \sigma_{3},
$$

are satisfied.

Proof. Under the hypothesis $\left(\boldsymbol{H}_{1}\right)$ and assuming that $q_{n} \neq\left(-1 / q_{r}^{*}\right)$, the four left-hand sides of (5.2) are continuous functions of $\zeta \in \mathbb{T}$ with the appropriate analytic continuation properties. Thus $\phi_{n}^{\text {up }}(z)$, $\bar{\phi}_{n}^{\mathrm{dn}}(z), \bar{\psi}_{n}^{\text {up }}(z)$, and $\psi_{n}^{\mathrm{dn}}(z)$ can be written as the above Fourier series in $\zeta$, where the corresponding Fourier coefficients are sequences in $\ell^{2}$. On the other hand, since $\gamma(z) / \zeta$ is continuous and nonzero for $|\zeta| \leq 1$ and $\zeta \gamma(z)$ is continuous and nonzero for $|\zeta| \geq 1$ (infinity included) [See Appendix B, last paragraph], the functions $\gamma(z)^{-1} \phi_{n}^{\text {dn }}(z), \gamma(z)^{-1} \bar{\phi}^{\text {up }}(z), \gamma(z)^{-1} \bar{\psi}^{\text {dn }}(z)$, and $\gamma(z)^{-1} \psi_{n}^{\text {up }}(z)$ are analytic on their domains of analyticity and continuous on their closures. Thus they can be expanded into the above Fourier series, where the coefficients belong to $\ell^{2}$. The symmetry relations (5.5) follow from (5.1) and (3.13).

Writing

$$
\boldsymbol{K}(n, s)=\left(\begin{array}{l}
K_{1}(n, s) K_{2}(n, s) \\
K_{3}(n, s) K_{4}(n, s)
\end{array}\right), \quad \boldsymbol{M}(n, s)=\left(\begin{array}{l}
M_{1}(n, s) M_{2}(n, s) \\
M_{3}(n, s) M_{4}(n, s)
\end{array}\right)
$$


and using that $\boldsymbol{K}(n, s)$ and $\boldsymbol{M}(n, s)$ have zero diagonal elements if $n-s$ is odd, and zero off-diagonal elements if $n-s$ is even [cf. (5.5)], we get

$$
\begin{aligned}
& \frac{\zeta^{-n} \phi_{n}^{\text {up }}(z)}{\left(1+A^{2}\right)^{\frac{n}{2}}}=\sum_{k=0}^{\infty}\left[\zeta^{-2 k} M_{1}(n, n-2 k)+\zeta^{-2 k-1} \gamma(z) q_{l}^{*} M_{2}(n, n-2 k-1)\right], \\
& \frac{\zeta^{-n} \phi_{n}^{\text {dn }}(z)}{\left(1+A^{2}\right)^{\frac{n}{2}}}=\sum_{k=0}^{\infty}\left[\zeta^{-2 k-1} M_{3}(n, n-2 k-1)+\zeta^{-2 k} \gamma(z) q_{l}^{*} M_{4}(n, n-2 k)\right], \\
& \frac{\zeta^{n} \bar{\phi}_{n}^{\text {up }}(z)}{\left(1+A^{2}\right)^{\frac{n}{2}}}=\sum_{k=0}^{\infty}\left[\zeta^{2 k} \gamma(z) q_{l} M_{1}(n, n-2 k)+\zeta^{2 k+1} M_{2}(n, n-2 k-1)\right], \\
& \frac{\zeta^{n} \bar{\phi}_{n}^{\text {dn }}(z)}{\left(1+A^{2}\right)^{\frac{n}{2}}}=\sum_{k=0}^{\infty}\left[\zeta^{2 k+1} \gamma(z) q_{l} M_{3}(n, n-2 k-1)+\zeta^{2 k} M_{4}(n, n-2 k)\right],
\end{aligned}
$$

where $M_{1}(n, n)=M_{4}(n, n)=1$. Here $\gamma(z)$ has the same $\zeta$-domain of analyticity as the left-hand side of the equation it appears in. Using (B.4a) in the second equation and (B.3a) in the third equation, we obtain on comparing the dominant terms

$$
\begin{aligned}
& M_{3}(n, n-1)=-\left(1+A^{2}\right)^{-1 / 2}\left[q_{n-1}^{*}-q_{l}^{*}\right], \\
& M_{2}(n, n-1)=\left(1+A^{2}\right)^{-1 / 2}\left[q_{n-1}-q_{l}\right] .
\end{aligned}
$$

Analogous expressions can be derived for the other two Jost solutions. This will lead to expressions for $K_{1}(n, n), K_{2}(n, n+1), K_{3}(n, n+1)$, and $K_{4}(n, n)$ in terms of the in principle unknown coefficients $\bar{C}_{s}^{\text {up }}, \bar{C}_{s}^{\mathrm{dn}}, C_{s}^{\mathrm{up}}$, and $C_{s}^{\mathrm{dn}}$. Indeed,

$$
\begin{aligned}
& \frac{\zeta^{-n} \bar{\psi}_{n}^{\mathrm{up}}(z)}{\left(1+A^{2}\right)^{\frac{n}{2}}}=\sum_{k=0}^{\infty}\left[\zeta^{2 k} K_{1}(n, n+2 k)+\zeta^{2 k+1} \gamma(z) q_{r}^{*} K_{2}(n, n+2 k+1)\right], \\
& \frac{\zeta^{-n} \bar{\psi}_{n}^{\mathrm{dn}}(z)}{\left(1+A^{2}\right)^{\frac{n}{2}}}=\sum_{k=0}^{\infty}\left[\zeta^{2 k+1} K_{3}(n, n+2 k+1)+\zeta^{2 k} \gamma(z) q_{r}^{*} K_{4}(n, n+2 k)\right], \\
& \frac{\zeta^{n} \psi_{n}^{\mathrm{up}}(z)}{\left(1+A^{2}\right)^{\frac{n}{2}}}=\sum_{k=0}^{\infty}\left[\zeta^{-2 k} \gamma(z) q_{r} K_{1}(n, n+2 k)+\zeta^{-2 k-1} K_{2}(n, n+2 k+1)\right], \\
& \frac{\zeta^{n} \psi_{n}^{\mathrm{dn}}(z)}{\left(1+A^{2}\right)^{\frac{n}{2}}}=\sum_{k=0}^{\infty}\left[\zeta^{-2 k-1} \gamma(z) q_{r} K_{3}(n, n+2 k+1)+\zeta^{-2 k} K_{4}(n, n+2 k)\right],
\end{aligned}
$$

where $\gamma(z)$ has the same $\zeta$-domain of analyticity as the left-hand side of the equation it appears in. Using (B.4a) in the second equation and (B.3a) in the third equation, we obtain on comparing the dominant terms

$$
\begin{aligned}
K_{1}(n, n) & =\bar{C}_{n}^{\mathrm{up}}, \\
K_{2}(n, n+1) & =C_{n}^{\mathrm{up}}+q_{r}\left(1+A^{2}\right)^{-1 / 2} \bar{C}_{n}^{\mathrm{up}}, \\
K_{3}(n, n+1) & =\bar{C}_{n}^{\mathrm{dn}}-q_{r}^{*}\left(1+A^{2}\right)^{-1 / 2} C_{n}^{\mathrm{dn}}, \\
K_{4}(n, n) & =C_{n}^{\mathrm{dn}} .
\end{aligned}
$$

\section{Marchenko equations}

In this section we derive the left Marchenko summation equations satisfied by $\boldsymbol{M}(n, m)$ starting from a Riemann-Hilbert problem satisfied by the Jost solutions. With the help of (5.7b) we can then 
determine the potential from their solutions. This is not possible for the right Marchenko equations satisfied by $\boldsymbol{K}(n, m)$, because (5.9) does not allow one to compute the potential from their solutions without apriori information on the potential. Throughout this section we assume that the discrete eigenvalues are algebraically simple in order to greatly simplify the derivation of the left Marchenko equations.

Rewriting (4.1) with the help of the reflection and transmission coefficients we obtain the Riemann-Hilbert problems

$$
\begin{aligned}
\left(\bar{\psi}_{n}(z) \bar{\phi}_{n}(z)\right) & =\left(\phi_{n}(z) \psi_{n}(z)\right) \sigma_{3} \boldsymbol{S}(z) \sigma_{3}, \\
\left(\phi_{n}(z) \psi_{n}(z)\right) & =\left(\bar{\psi}_{n}(z) \bar{\phi}_{n}(z)\right) \sigma_{3} \overline{\boldsymbol{S}}(z) \sigma_{3},
\end{aligned}
$$

where

$$
\boldsymbol{S}(z)=\left(\begin{array}{cc}
a(z)^{-1} & r(z) \\
\rho(z) & c(z)^{-1}
\end{array}\right), \quad \overline{\boldsymbol{S}}(z)=\left(\begin{array}{cc}
\bar{c}(z)^{-1} & \bar{\rho}(z) \\
\bar{r}(z) & \bar{a}(z)^{-1}
\end{array}\right)
$$

are the scattering matrix and its inverse.

Let us write the second Riemann-Hilbert problem (6.1b) as follows:

$$
\begin{gathered}
\zeta^{-n} \phi_{n}(z)=\frac{\zeta^{-n} \bar{\psi}_{n}(z)}{\bar{c}(z)}-\zeta^{-2 n} \bar{r}(z) \zeta^{n} \bar{\phi}_{n}(z) \\
\zeta^{n} \bar{\phi}_{n}(z)=\frac{\zeta^{n} \psi_{n}(z)}{c(z)}-\zeta^{2 n} r(z) \zeta^{-n} \phi_{n}(z)
\end{gathered}
$$

where the left-hand side of (6.2a) [(6.2b)] is continuous in $\zeta \in \overline{\Lambda^{\mp}}$ and analytic in $\zeta \in \Lambda^{\mp}$ with the finite limit $\left(1+A^{2}\right)^{\frac{n}{2}}\left(\begin{array}{l}1 \\ 0\end{array}\right)$ at infinity $\left[\left(1+A^{2}\right)^{\frac{n}{2}}\left(\begin{array}{l}0 \\ 1\end{array}\right)\right.$ at zero] and the right-hand side of $(6.2 \mathrm{a})$ [(6.2b)] is continuous in $\zeta \in \overline{\Lambda^{ \pm}}$and meromorphic in $\zeta \in \Lambda^{ \pm}$with a finite limit at zero [infinity]. Writing

$$
\frac{1}{\bar{c}(z)}=\bar{t}_{l}^{0}(\zeta)+\sum_{k} \frac{\hat{\bar{\tau}}_{k}}{\zeta-\zeta\left(\bar{z}_{k}\right)}, \quad \frac{1}{c(z)}=t_{l}^{0}(\zeta)+\sum_{k} \frac{\hat{\tau}_{k}}{\zeta-\zeta\left(z_{k}\right)}
$$

where $\bar{t}_{l}^{0}(\zeta)\left[t_{l}^{0}(\zeta)\right]$ is continuous in $\zeta \in \overline{\Lambda^{ \pm}}$and analytic in $\zeta \in \Lambda^{ \pm}$, we obtain for $m \leq n-1$ with the help of (4.14c) and (4.14d)

$$
\begin{gathered}
\oint \frac{d \zeta}{2 \pi i} \zeta^{-m-1} \phi_{n}(z)=+\sum_{k} \zeta\left(\bar{z}_{k}\right)^{-m-1} \bar{\phi}_{n}\left(\bar{z}_{k}\right) \bar{D}_{k}-\oint \frac{d \zeta}{2 \pi i} \zeta^{-m-1} \bar{r}(z) \bar{\phi}_{n}(z), \\
\oint \frac{d \zeta}{2 \pi i} \zeta^{m-1} \bar{\phi}_{n}(z)=-\sum_{k} \zeta\left(z_{k}\right)^{m-1} \phi_{n}\left(z_{k}\right) D_{k}-\oint \frac{d \zeta}{2 \pi i} \zeta^{m-1} r(z) \phi_{n}(z),
\end{gathered}
$$

where we integrate with respect to $\zeta \in \mathbb{T}$ in the counterclockwise sense. Note that the left-hand side and the first term on the right-hand side do not contain any contribution at the (possible) pole at $\zeta=0[\zeta=\infty]$ if $m \leq n-1$. Dividing by $\left(1+A^{2}\right)^{\frac{n}{2}}$ and using (5.2c) and (5.2d) we arrive at the 
equations

$$
\begin{aligned}
\sum_{s=-\infty}^{n} \boldsymbol{M}(n, s) \oint \frac{d \zeta}{2 \pi i} \zeta^{s-m-1}\left(\begin{array}{c}
1 \\
\gamma(z) q_{l}^{*}
\end{array}\right) & =-\sum_{s=-\infty}^{n} \boldsymbol{M}(n, s) \bar{F}(s+m), \\
\sum_{s=-\infty}^{n} \boldsymbol{M}(n, s) \oint \frac{d \zeta}{2 \pi i} \zeta^{m-s-1}\left(\begin{array}{c}
\gamma(z) q_{l} \\
1
\end{array}\right) & =-\sum_{s=-\infty}^{n} \boldsymbol{M}(n, s) F(s+m),
\end{aligned}
$$

where the left Marchenko kernels are given by

$$
\begin{aligned}
& \bar{F}(j)=-\sum_{k} \zeta\left(\bar{z}_{k}\right)^{-j-1} \bar{D}_{k}\left(\begin{array}{c}
\gamma\left(\bar{z}_{k}\right) q_{l} \\
1
\end{array}\right)+\oint \frac{d \zeta}{2 \pi i} \zeta^{-j-1} \bar{r}(z)\left(\begin{array}{c}
\gamma(z) q_{l} \\
1
\end{array}\right), \\
& F(j)=+\sum_{k} \zeta\left(z_{k}\right)^{j-1} D_{k}\left(\begin{array}{c}
1 \\
\gamma\left(z_{k}\right) q_{l}^{*}
\end{array}\right)+\oint \frac{d \zeta}{2 \pi i} \zeta^{j-1} r(z)\left(\begin{array}{c}
1 \\
\gamma(z) q_{l}^{*}
\end{array}\right) .
\end{aligned}
$$

In (6.3a) $[(6.3 \mathrm{~b})] \gamma(z)$ is analytic in $\zeta \in \Lambda^{\mp}$. Putting

$$
\gamma(z)= \begin{cases}-\sum_{\sigma=0}^{\infty} \zeta^{-\sigma-1} \gamma_{l, \sigma}, & |\zeta|>1, \\ +\sum_{\sigma=0}^{\infty} \zeta^{\sigma+1} \gamma_{l, \sigma}, & |\zeta|<1,\end{cases}
$$

where $\gamma_{l, \sigma}=0$ if $\sigma$ is odd, (6.3) can be rewritten as

$$
\begin{aligned}
\boldsymbol{M}(n, m) & +\boldsymbol{F}(n+m)+\sum_{s=-\infty}^{n-1} \boldsymbol{M}(n, s) \boldsymbol{F}(s+m) \\
& +\sum_{s=m+1}^{n-1} \gamma_{l, s-m-1} \boldsymbol{M}(n, s) \boldsymbol{q}_{l} \sigma_{1}+\gamma_{l, n-m-1} \boldsymbol{q}_{l} \sigma_{1}=0_{2 \times 2},
\end{aligned}
$$

where

$$
\boldsymbol{F}(j)=(\bar{F}(j) F(j))=\left(\begin{array}{ll}
F_{1}(j) & F_{2}(j) \\
F_{3}(j) & F_{4}(j)
\end{array}\right) .
$$

Equations (3.8b) and (4.13) imply that

$$
r\left(z^{*-1}\right)^{*} \gamma\left(z^{*-1}\right)^{*}=\bar{r}(z) \gamma(z) .
$$

Moreover, $\zeta\left(\bar{z}_{k}\right)^{*}=\zeta\left(z_{k}\right)^{-1}$. Using (4.17) also, the Marchenko kernel satisfies the conjugation symmetry relation

$$
\boldsymbol{F}(j)^{*}=\sigma_{2} \boldsymbol{F}(j) \sigma_{2} .
$$

Since its diagonal entries are nonzero, we do not get the familiar identity $\boldsymbol{F}(j)^{\dagger}=\sigma_{3} \boldsymbol{F}(j) \sigma_{3}$ valid in the vanishing case [12].

Using (4.15) plus the fact that $r(z), \bar{r}(z)$, and $\gamma(z)$ are odd functions of $\zeta$, we obtain from (6.4) the parity symmetry

$$
\boldsymbol{F}(j)=(-1)^{j} \sigma_{3} \boldsymbol{F}(j) \sigma_{3} .
$$

To write the Marchenko equation (6.6) in a more familiar fashion, we observe that the matrices $\boldsymbol{M}(n, m)$ and $\boldsymbol{F}(n+m)$ are diagonal if $n-m$ is even and off-diagonal if $n-m$ is odd [cf. (5.5)]. We also observe that $\boldsymbol{q}_{l} \sigma_{1}$ is a diagonal matrix and that $\gamma_{l, s-m-1}$ is nonzero iff $s-m$ is odd. On 
one hand, if $n-m$ is even, then $\boldsymbol{M}(n, m)$ and $\boldsymbol{F}(n+m)$ are diagonal and $s-n$ is to be odd and hence $\boldsymbol{M}(n, s) \boldsymbol{q}_{l} \sigma_{1}$ is to be off-diagonal for $\gamma_{l, s-m-1}$ to be nonzero. Thus the finite sum in (6.6) must vanish. On the other hand, if $n-m$ is odd, then $\boldsymbol{M}(n, m)$ and $\boldsymbol{F}(n+m)$ are off-diagonal and $s-n$ is to be even and hence $\boldsymbol{M}(n, s) \boldsymbol{q}_{l} \sigma_{1}$ is to be diagonal for $\gamma_{l, s-m-1}$ to be nonzero. Thus the finite sum in (6.6) must vanish. In either case, the finite sum in (6.6) vanishes. As a result, (6.6) is reduced to the more familiarly looking Marchenko equation (cf. [32, (4.16)-(4.17)])

$$
\boldsymbol{M}(n, m)+\boldsymbol{F}(n+m)+\sum_{s=-\infty}^{n-1} \boldsymbol{M}(n, s) \boldsymbol{F}(s+m)=-\gamma_{l, n-m-1} \boldsymbol{q}_{l} \sigma_{1} .
$$

Writing $m=n-2 k-2$ for the diagonal entries and $m=n-2 k-1$ for the off-diagonal entries and substituting $s=n-2 j-2$ on the diagonal and $s=n-2 j-1$ off-diagonal, we obtain from (6.9) the coupled scalar Marchenko equations Using that $\gamma_{l, 2[k-j]-1}=\gamma_{l, 2 k+1}=0$, we finally arrive at the left Marchenko equations

$$
\begin{aligned}
M_{1}(n, n & \left.-2 k-2)+F_{1}(2[n-k-1])\right) \\
& +\sum_{j=0}^{\infty}\left\{M_{1}(n, n-2 j-2) F_{1}(2[n-j-k-2])\right. \\
& \left.+M_{2}(n, n-2 j-1) F_{3}(2[n-j-k]-3)\right\}=0, \\
M_{2}(n, n- & \left.2 k-1)+F_{2}(2[n-k]-1)\right) \\
& +\sum_{j=0}^{\infty}\left\{M_{1}(n, n-2 j-2) F_{2}(2[n-j-k]-3)\right. \\
& \left.+M_{2}(n, n-2 j-1) F_{4}(2[n-j-k-1])\right\}-q_{l}^{*} \gamma_{l, 2 k}=0, \\
M_{3}(n, n & -2 k-1)+F_{3}(2[n-k]-1) \\
& +\sum_{j=0}^{\infty}\left\{M_{4}(n, n-2 j-2) F_{3}(2[n-j-k]-3)\right. \\
& \left.+M_{3}(n, n-2 j-1) F_{1}(2[n-j-k-1])\right\}+q_{l} \gamma_{l, 2 k}=0, \\
M_{4}(n, n & \left.-2 k-2)+F_{4}(2[n-k-1])\right) \\
& +\sum_{j=0}^{\infty}\left\{M_{4}(n, n-2 j-2) F_{4}(2[n-j-k-2])\right. \\
& \left.+M_{3}(n, n-2 j-1) F_{2}(2[n-j-k]-3)\right\}=0,
\end{aligned}
$$

where the coefficients $\gamma_{l, 2 k}$ are computed in the last paragraph of Appendix C. Note that we have arrived at two systems of two coupled equations.

We now compute the one-soliton solution by solving the Marchenko equations (6.10a)-(6.10b) and using the time evolution of the asymptotic potential values and the norming constants.

Example 6.1 (One-soliton solution). In this example the reflection coefficients vanish and there are four discrete eigevalues $\left\{ \pm z_{1}, \pm \bar{z}_{1}\right\}$, where $\bar{z}_{1}=z_{1}^{*-1}$. The norming constants satisfy

$$
D_{1}=D_{-1}, \quad \bar{D}_{1}=\bar{D}_{-1}=\zeta_{1}^{*-2} D_{1}^{*}
$$


where $\zeta_{1}=\zeta\left(z_{1}\right)$. We also put $\gamma_{1}=\gamma\left(z_{1}\right)$. It is then easily verified that

$$
\begin{array}{lll}
\boldsymbol{F}(j) & =2\left(\begin{array}{cc}
\zeta_{1}^{* j-1} D_{1}^{*} \gamma_{1}^{*} q_{l} & 0 \\
0 & \zeta_{1}^{j-1} D_{1} \gamma_{1} q_{l}^{*}
\end{array}\right), & j \text { even }, \\
\boldsymbol{F}(j) & =2\left(\begin{array}{cc}
0 & \zeta_{1}^{j-1} D_{1} \\
-\zeta_{1}^{* j-1} D_{1}^{*} & 0
\end{array}\right), & j \text { odd },
\end{array}
$$

which satisfies the symmetry relations (6.7)-(6.8). Then (6.10a)-(6.10b) imply that

$$
\begin{aligned}
& M_{1}(n, n-2 k-2)=-2 \zeta_{1}^{* 2[n-k]-3} D_{1}^{*} \gamma_{1}^{*} q_{l}-2 \zeta_{1}^{* 2[n-k]-5} D_{1}^{*} L^{\mathrm{up}}(n), \\
& M_{2}(n, n-2 k-1)=\gamma_{l, 2 k} q_{l}^{*}-2 \zeta_{1}^{2[n-k-1]} D_{1}-2 \zeta_{1}^{2[n-k-2]} D_{1} L^{\mathrm{dn}}(n),
\end{aligned}
$$

where

$$
\begin{aligned}
& L^{\mathrm{up}}(n)=\sum_{j=0}^{\infty}\left\{M_{1}(n, n-2 j-2) \zeta_{1}^{*-2 j} \gamma_{1}^{*} q_{l}-M_{2}(n, n-2 j-1) \zeta_{1}^{*-2 j+1}\right\}, \\
& L^{\mathrm{dn}}(n)=\sum_{j=0}^{\infty}\left\{M_{1}(n, n-2 j-2) \zeta_{1}^{-2 j}+M_{2}(n, n-2 j-1) \zeta_{1}^{-2 j+1} \gamma_{1} q_{l}^{*}\right\} .
\end{aligned}
$$

Using sums of geometric series of ratio $\zeta_{1}^{-4}, \zeta_{1}^{*-4}$, or $\left|\zeta_{1}\right|^{-4}$, we arrive at the following linear system

$$
\left(\begin{array}{cc}
1+\frac{2 D_{1}^{*} \gamma_{1}^{*} q \zeta_{1}^{* 2 n-5}}{1-\zeta_{1}^{*-4}} & -\frac{2 D_{1} \zeta_{1}^{*} \zeta_{1}^{2 n-4}}{1-\left|\zeta_{1}\right|^{-4}} \\
\frac{2 D_{1}^{*} \zeta_{1}^{* 2 n-5}}{1-\left|\zeta_{1}\right|^{-4}} & 1+\frac{2 D_{1} \gamma_{1} q_{1}^{*} \zeta_{1}^{2 n-3}}{1-\zeta_{1}^{-4}}
\end{array}\right)\left(\begin{array}{l}
L^{\mathrm{up}}(n) \\
L^{\mathrm{dn}}(n)
\end{array}\right)=\left(\begin{array}{l}
R^{\mathrm{up}}(n) \\
R^{\mathrm{dn}}(n)
\end{array}\right),
$$

where

$$
\begin{aligned}
& R^{\mathrm{up}}(n)=-q_{l}^{*} \zeta_{1}^{*} \Gamma_{l}\left(\zeta_{1}^{*-1}\right)+\frac{2 D_{1} \zeta_{1}^{*} \zeta_{1}^{2 n-2}}{1-\left|\zeta_{1}\right|^{-4}}-\frac{2 D_{1}^{*} \gamma_{1}^{* 2} q_{l}^{2} \zeta_{1}^{* 2 n-3}}{1-\zeta_{1}^{*-4}} \\
& R^{\mathrm{dn}}(n)=\gamma_{1} q_{l}^{* 2} \zeta_{1} \Gamma_{l}\left(\zeta_{1}^{-1}\right)-\frac{2 D_{1} \gamma_{1} q_{l}^{*} \zeta_{1}^{2 n-1}}{1-\zeta_{1}^{-4}}-\frac{2 D_{1}^{*} \gamma_{1}^{*} q_{l} \zeta_{1}^{* 2 n-3}}{1-\left|\zeta_{1}\right|^{-4}}
\end{aligned}
$$

Here $\Gamma_{l}(w)$ is defined in Appendix C. The one-soliton solution is obtained by applying (5.7b) while replacing $q_{l}$ by $e^{-2 i A^{2} \tau} q_{l}$ [see (7.3) below] and $D_{1}$ by $e^{-2 i \omega\left(z_{1}\right) \tau} D_{1}$ [see (7.7b) below].

\section{Time evolution of the scattering data}

The integrable discrete nonlinear Schrödinger (IDNLS) equation (1.3) is the so-called zero curvature condition for the coupled linear system $[5,16,30,31]$

$$
\begin{aligned}
V_{n+1} & =\left(\boldsymbol{Z}+\boldsymbol{q}_{n}\right) V_{n}, \\
\frac{d}{d \tau} V_{n} & =\boldsymbol{T}_{n}(z ; \tau) V_{n} \\
& =-i \sigma_{3}\left\{\frac{1}{2}\left(\boldsymbol{Z}-\boldsymbol{Z}^{-1}\right)^{2}-\boldsymbol{q}_{n} \boldsymbol{q}_{n-1}+\boldsymbol{Z q}_{n}-\boldsymbol{Z}^{-1} \boldsymbol{q}_{n-1}\right\} V_{n} .
\end{aligned}
$$

where $V_{n}(z ; \tau)$ is a suitable invertible matrix function. Then for any other invertible matrix solution $W_{n}(z ; \tau)$ to (7.1a) there exists a nonsingular matrix $C_{W}(z ; \tau)$ such that $W_{n}(z ; \tau)=V_{n}(z ; \tau) C_{W}(z ; \tau)^{-1}$ 
and hence

$$
\frac{d}{d \tau} W_{n}=\boldsymbol{T}_{n} W_{n}-W_{n}\left[C_{W}\right]_{\tau} C_{W}^{-1} .
$$

For $W_{n}$ we shall henceforth choose the two Jost matrices $\Psi_{n}$ and $\Phi_{n}$ which are related by $\Phi_{n}=\Psi_{n} S$. We then get

$$
\begin{aligned}
S_{\tau} & =\Psi_{n}^{-1}\left[\Phi_{n}\right]_{\tau}-\Psi_{n}^{-1}\left[\Psi_{n}\right]_{\tau} \Psi_{n}^{-1} \Phi_{n}=\Psi_{n}^{-1}\left[\Phi_{n}\right]_{\tau}-\Psi_{n}^{-1}\left[\Psi_{n}\right]_{\tau} S \\
& =\Psi_{n}^{-1}\left(T_{n} \Phi_{n}-\Phi_{n}\left[C_{\Phi}\right]_{\tau} C_{\Phi}^{-1}\right)-\Psi_{n}^{-1}\left(T_{n} \Psi_{n}-\Psi_{n}\left[C_{\Psi}\right]_{\tau} C_{\Psi}^{-1}\right) S \\
& =\left[C_{\Psi}\right]_{\tau} C_{\Psi}^{-1} S-S\left[C_{\Phi}\right]_{\tau} C_{\Phi}^{-1} .
\end{aligned}
$$

Differentiating (7.1a) with respect to $\tau$ and using (7.1b) with $n$ replaced by $n+1$ as well as the invertibility of $V_{n}(z ; \tau)$, we obtain the IDNLS equation

$$
i \sigma_{3} \frac{d}{d \tau} \boldsymbol{q}_{n}=\boldsymbol{q}_{n+1}-2 \boldsymbol{q}_{n}+\boldsymbol{q}_{n-1}-\boldsymbol{q}_{n+1} \boldsymbol{q}_{n}^{2}-\boldsymbol{q}_{n}^{2} \boldsymbol{q}_{n-1},
$$

in agreement with (1.3). Taking the limit of $\boldsymbol{T}_{n}(z ; \tau)$ as $n \rightarrow \pm \infty$, we obtain

$$
\boldsymbol{T}_{r / l}(z)=-i \sigma_{3}\left\{\frac{1}{2}\left(\boldsymbol{Z}-\boldsymbol{Z}^{-1}\right)^{2}-\boldsymbol{q}_{r / l}(\tau)^{2}+\left(\boldsymbol{Z}-\boldsymbol{Z}^{-1}\right) \boldsymbol{q}_{r / l}(\tau)\right\} .
$$

Taking the same limit in (7.3), we obtain

$$
i \sigma_{3} \frac{d}{d \tau} \boldsymbol{q}_{r / l}(\tau)=-2 \boldsymbol{q}_{r / l}(\tau)^{3}=2 A^{2} \boldsymbol{q}_{r / l}(\tau) .
$$

Taking for $W_{n}$ the Jost matrices $\Psi_{n}$ and $\Phi_{n}$ so that

$$
\begin{array}{ll}
\Psi_{n}(z) \simeq\left(\boldsymbol{Z}+\boldsymbol{q}_{r}\right) W_{r}(z)=\left(1+A^{2}\right)^{\frac{n}{2}} W_{r}(z) \mathscr{Z}^{n}, & n \rightarrow+\infty, \\
\Phi_{n}(z) \simeq\left(\boldsymbol{Z}+\boldsymbol{q}_{l}\right) W_{l}(z)=\left(1+A^{2}\right)^{\frac{n}{2}} W_{l}(z) \mathscr{Z}^{n}, & n \rightarrow-\infty,
\end{array}
$$

where $\mathscr{Z}=\operatorname{diag}\left(\zeta, \zeta^{-1}\right)$, we obtain after some effort [See Appendix D]

$$
\left[C_{\Psi}\right]_{\tau} C_{\Psi}^{-1}=\left[C_{\Phi}\right]_{\tau} C_{\Phi}^{-1}=-i \omega(z) \sigma_{3},
$$

where

$$
\omega(z)=A^{2}+\frac{1}{2}\left(z-z^{-1}\right)\left(\zeta-\zeta^{-1}\right)
$$

As a result,

$$
S_{\tau}=-i \omega(z)\left\{\sigma_{3} S-S \sigma_{3}\right\}
$$

implying that

$$
S(z ; \tau)=e^{-i \omega(z) \tau \sigma_{3}} S(z ; 0) e^{i \omega(z) \tau \sigma_{3}} .
$$

We finally arrive at the following time evolution of the scattering coefficients:

$$
\begin{array}{llrl}
a(z ; \tau) & =a(z ; 0), & \bar{a}(z ; \tau)=\bar{a}(z ; 0), \\
b(z ; \tau)=e^{2 i \omega(z) \tau} b(z ; 0), & \bar{b}(z ; \tau)=e^{-2 i \omega(z) \tau} \bar{b}(z ; 0) .
\end{array}
$$


Using (4.11) and (4.12) we obtain the following time evolution for the reflection coefficients:

$$
\begin{aligned}
\rho(z ; \tau) & =e^{2 i \omega(z) \tau} \rho(z ; 0), & & \bar{\rho}(z ; \tau)=e^{-2 i \omega(z) \tau} \bar{\rho}(z ; 0) . \\
r(z ; \tau) & =e^{-2 i \omega(z) \tau} r(z ; 0), & & \bar{r}(z ; \tau)=e^{2 i \omega(z) \tau} \bar{r}(z ; 0) .
\end{aligned}
$$

Let us now determine the time evolution of the norming constants. Differentiating (4.14a) with respect to $\tau$ and using that

$$
\begin{aligned}
{\left[\psi_{n}\left(z_{k}\right)\right]_{\tau} } & =\left\{T_{n}\left(z_{k}\right)+i \omega\left(z_{k}\right)\right\} \psi_{n}\left(z_{k}\right), \\
{\left[\phi_{n}\left(z_{k}\right)\right]_{\tau} } & =\left\{T_{n}\left(z_{k}\right)-i \omega\left(z_{k}\right)\right\} \phi_{n}\left(z_{k}\right),
\end{aligned}
$$

we obtain

$$
\left[T_{n}\left(z_{k}\right)+i \omega\left(z_{k}\right)\right] \psi_{n}\left(z_{k}\right) C_{k}+\psi_{n}\left(z_{k}\right)\left[C_{n}\right]_{\tau}=\left[T_{n}\left(z_{k}\right)-i \omega\left(z_{k}\right)\right] \phi_{n}\left(z_{k}\right) \tau_{k}
$$

where we have used that $\tau_{k}$ does not depend on $\tau$. We thus get $\left[C_{k}\right]_{\tau}=2 i \omega\left(z_{k}\right) C_{k}$ and hence $C_{k}(\tau)=$ $e^{2 i \omega\left(z_{k}\right) \tau} C_{k}(0)$. In analogous ways we therefore arrive at the following four identities:

$$
\begin{aligned}
C_{k}(\tau) & =e^{2 i \omega\left(z_{k}\right) \tau} C_{k}(0), & \bar{C}_{k}(\tau) & =e^{-2 i \omega\left(\bar{z}_{k}\right) \tau} \bar{C}_{k}(0), \\
D_{k}(\tau) & =e^{-2 i \omega\left(z_{k}\right) \tau} D_{k}(0), & & \bar{D}_{k}(\tau)=e^{2 i \omega\left(\bar{z}_{k}\right) \tau} \bar{D}_{k}(0) .
\end{aligned}
$$

When numbering the discrete eigenvalues as we have done in Section 4 to account for parity and circle conjugation symmetry, we obtain

$$
\omega\left(z_{-k}\right)=\omega\left(z_{k}\right), \quad \omega\left(\bar{z}_{k}\right)=\omega\left(z_{k}\right)^{*},
$$

while $\zeta\left(\bar{z}_{k}\right)=\zeta\left(z_{k}\right)^{*-1}$.

\section{Acknowledgments}

The author is greatly indebted to Barbara Prinari for a communication regarding the time evolution of the scattering data and to Francesco Demontis and the anonymous referee for their comments on the manuscript. The research leading to this article was supported in part by INdAM-GNFM.

\section{References}

[1] M.J. Ablowitz, G. Biondini, and B. Prinari, Inverse scattering transform for the integrable discrete nonlinear Schrödinger equation with nonvanishing boundary conditions, Inverse Problems 23, 17111758 (2007).

[2] M.J. Ablowitz and J.F. Ladik, Nonlinear differential-difference equations, J. Math. Phys. 16, 598-603 (1975).

[3] M.J. Ablowitz and J.F. Ladik, A nonlinear difference scheme and inverse scattering, Stud. Appl. Math. 55, 213-229 (1976).

[4] M.J. Ablowitz and J.F. Ladik, Nonlinear differential-difference equations and nonlinearity, J. Math. Phys. 17, 1011-1018 (1976).

[5] M.J. Ablowitz, B. Prinari, and A.D. Trubatch, Discrete and Continuous Nonlinear Schrödinger Systems, Cambridge University Press, Cambridge, 2004.

[6] M.J. Ablowitz and H. Segur, Solitons and the Inverse Scattering Transform, SIAM, Philadelphia, 1981.

[7] R. Beals and R. Wong, Special Functions, Cambridge Univ. Press, Cambridge, 2010.

[8] G. Biondini and G. Kovačić, Inverse scattering transform for the focusing nonlinear Schrödinger equation with nonzero boundary conditions, J. Math. Phys. 55, 031506 (2014), 22 pp. 
[9] D. Cai, A.P. Bishop, and N. Grønbech-Jensen, Spatially localized, temporally quasi-periodic, discrete nonlinear excitations, Phys. Rev. E 52, 5784-5787 (1995).

[10] F. Demontis, B. Prinari, C. van der Mee, and F. Vitale, The inverse scattering transform for the defocusing nonlinear Schrödinger equation with nonzero boundary conditions, Stud. Appl. Math. 131, 1-40 (2013).

[11] F. Demontis, B. Prinari, C. van der Mee, and F. Vitale, The inverse scattering transform for the focusing nonlinear Schrödinger equation with asymmetric boundary conditions, J. Math. Phys. 55, 101505 (2015).

[12] F. Demontis and C. van der Mee, Exact solutions to the integrable discrete nonlinear Schrödinger equation under a quasiscalarity condition, Commun. Appl. Ind. Math. 2(2), 2011, e-372, 21 pp.

[13] F. Demontis and C. van der Mee, Closed form solutions to the integrable discrete nonlinear Schrödinger equation, J. Nonlin. Math. Phys. 19(2) (2012), 1250010, 22 pp.

[14] F. Demontis and C. van der Mee, An alternative approach to integrable discrete nonlinear Schrödinger equations, Acta Appl. Math. 131, 29-47 (2014).

[15] A. Doliwa and P.M. Santini, Integrable dynamics of a discrete curve and the Ablowitz-Ladik hierarchy, J. Math. Phys. 36, 1259-1273 (1995).

[16] L.D. Faddeev and L.A. Takhtajan, Hamiltonian Methods in the Theory of Solitons, Classics in Mathematics, Springer, New York, 1987.

[17] V.S. Gerdjikov and M.I. Ivanov, Block discrete Zakharov-Shabat systems. I. Generalized Fourier expansion, United Nuclear Research Institute Report E2-81-811, Dubna, 1981.

[18] V.S. Gerdjikov and M.I. Ivanov, Block discrete Zakharov-Shabat systems. II. Hamiltonian structures, United Nuclear Research Institute Report E2-81-812, Dubna, 1981.

[19] A. Hasegawa and M. Matsumoto, Optical Solitons in Fibers, 3rd ed., Springer, Berlin, 2002.

[20] A. Hasegawa and F. Tappert, Transmission of stationary nonlinear optical pulses in dispersive dielectric fibers. I. Anomalous dispersion, Appl. Phys. Lett. 23, 142-144 (1973).

[21] M. Hisakado and M. Wadati, Integrable dynamics of discrete surfaces. II, J. Phys. Soc. Japan 65, 389393 (1996).

[22] Y. Ishimori, An integrable classical spin chain, J. Phys. Soc. Japan 51, 3417-3418 (1982).

[23] V.M. Kenkre and D.K. Campbell, Self-trapping on a dimer: Time-dependent solutions of a discrete nonlinear Schrödinger equation, Phys. Rev. B 34, 4959-4961 (1986).

[24] P.P. Kulish, Quantum difference nonlinear Schrödinger equation, Lett. Math. Phys. 5, 191-197 (1981).

[25] V. Lakshmikantham and D. Trigiante, Theory of Difference Equations: Numerical Methods and Applications, Marcel Dekker, New York, 2002.

[26] S.P. Novikov, S.V. Manakov, L.B. Pitaevskii, and V.E. Zakharov, Theory of Solitons. The Inverse Scattering Method, Plenum Press, New York, 1984.

[27] N. Papanicolau, Complete integrability for a discrete Heisenberg chain, J. Phys. A.: Math. Gen. 20, 3637-3652 (1987).

[28] Y.B. Suris, A note on an integrable discretization of the nonlinear Schrödinger equation, Inverse Problems 13, 1121-1136 (1997).

[29] S. Takeno and K. Hori, A propagating self-localized mode in a one-dimensional lattice with quartic anharmonicity, J. Phys. Soc. Japan 59, 3037-3040 (1990).

[30] T. Tsuchida, H. Ujino, and M. Wadati, Integrable semi-discretization of the coupled nonlinear Schrödinger equations, J. Phys. A 32 (1999), 2239-2262.

[31] C. van der Mee, Nonlinear Evolution Models of Integrable Type, SIMAI e-Lecture Notes 11, SIMAI, Torino, 2013.

[32] V.E. Vekslerchik and V.V. Konotop, Discrete nonlinear Schrödinger equation under nonvanishing boundary conditions, Inverse Problems 8, 889-909 (1992).

[33] V. E. Zakharov and A.B. Shabat, Exact theory of two-dimensional self-focusing and one dimensional self-modulation of waves in nonlinear media, Sov. Phys. JETP 34, 62-69 (1972). 


\section{Appendix A. Discrete Gronwall inequality}

Let $\left\{b_{k}\right\}_{k=n}^{\infty}$ be a sequence of nonnegative numbers. Then in [5, Lemma A.2] the following fundamental equality has been established:

$$
\sum_{k=n}^{\infty} b_{k}\left(\sum_{j=k+1}^{\infty} b_{j}\right)^{m}=\frac{1}{m+1}\left(\sum_{j=n}^{\infty} b_{j}\right)^{m+1}-B_{n}^{(m)}
$$

where $B_{n}^{(m)} \geq B_{n+1}^{(m)} \geq B_{n+2}^{(m)} \geq \ldots \geq 0$.

Although the general form of the discrete Gronwall inequality is well-known [25, Corollary 1.6.2], here we apply (A.1) to prove the version needed. The proof also supplies the convergence of iterating (A.2).

Proposition A.1. Suppose $\left\{p_{k}\right\}_{k=n}^{\infty}$ and $\left\{q_{k}\right\}_{k=n}^{\infty}$ are sequences of nonnegative numbers such that the series $\sum_{k=n+1}^{\infty} q_{k}$ converges and

$$
0 \leq p_{n} \leq 1+\sum_{k=n+1}^{\infty} q_{k} p_{k}
$$

Then

$$
0 \leq p_{n} \leq \exp \left(\sum_{k=n+1}^{\infty} q_{k}\right) .
$$

Proof. Iterating (A.2) we get (A.3) as follows:

$$
\begin{aligned}
p_{n} & \leq 1+\sum_{s=1}^{\infty} \sum_{k_{1}=n+1}^{\infty} q_{k_{1}} \sum_{k_{2}=k_{1}+1}^{\infty} q_{k_{2}} \sum_{k_{3}=k_{2}+1}^{\infty} q_{k_{3}} \ldots \sum_{k_{s}=k_{s-1}+1}^{\infty} q_{k_{s}} \\
& \leq 1+\sum_{s=1}^{\infty} \frac{1}{s !}\left(\sum_{k=n+1}^{\infty} q_{k}\right)^{s} \leq \exp \left(\sum_{k=n+1}^{\infty} q_{k}\right),
\end{aligned}
$$

where (A.1) has been used repeatedly.

In the same way we prove

Proposition A.2. Let $\left\{p_{k}\right\}_{k=-\infty}^{n}$ and $\left\{q_{k}\right\}_{k=-\infty}^{n}$ be sequences of nonnegative numbers such that the series $\sum_{k=-\infty}^{n-1} q_{k}$ converges and

$$
0 \leq p_{n} \leq 1+\sum_{k=-\infty}^{n-1} q_{k} p_{k}
$$

Then

$$
0 \leq p_{n} \leq \exp \left(\sum_{k=-\infty}^{n-1} q_{k}\right)
$$




\section{Appendix B. Conformal transformations}

In this appendix we derive the asymptotic behavior of the conformal transformation $\zeta(z)$ as $z \rightarrow 0$ and as $z \rightarrow \infty$. Indeed, for $0<|z|<1$ and $z \notin \Sigma$ we compute

$$
\begin{aligned}
\frac{d \zeta}{d z} & =\frac{1-z^{-2}}{2 \sqrt{1+A^{2}}}\left\{1-\frac{\frac{z+z^{-1}}{2 \sqrt{1+A^{2}}}}{\sqrt{\left(\frac{z+z^{-1}}{2 \sqrt{1+A^{2}}}\right)^{2}-1}}\right\} \\
& =\frac{z^{2}-1}{2 z^{2} \sqrt{1+A^{2}}} \frac{\sqrt{\left(z^{2}+1\right)^{2}-4\left(1+A^{2}\right) z^{2}}-\left(z^{2}+1\right)}{\sqrt{\left(z^{2}+1\right)^{2}-4\left(1+A^{2}\right) z^{2}}} \\
& =\frac{2\left(1-z^{2}\right) \sqrt{1+A^{2}}}{\left(z^{2}+1\right)^{2}-4\left(1+A^{2}\right) z^{2}+\left(z^{2}+1\right) \sqrt{\left(z^{2}+1\right)^{2}-4\left(1+A^{2}\right) z^{2}}}
\end{aligned}
$$

which tends to $\sqrt{1+A^{2}}$ as $z \rightarrow 0$. More precisely,

$$
\zeta(z)=z \sqrt{1+A^{2}}\left[z+\frac{1}{3}\left(1+\frac{3}{2} A^{2}\right) z^{2}+O\left(z^{4}\right)\right]
$$

as $z \rightarrow 0$. Using that $\zeta(1 / z)=[1 / \zeta(z)]$, we obtain

$$
\zeta(z)=\frac{z}{\sqrt{1+A^{2}}}\left[1-\frac{1}{3}\left(1+\frac{3}{2} A^{2}\right) z^{-2}+O\left(z^{-4}\right)\right]
$$

as $z \rightarrow \infty$.

We now obtain as $z \rightarrow 0$

$$
\begin{aligned}
\gamma(z) & =\frac{\zeta \sqrt{1+A^{2}}-z}{A^{2}}=z+O\left(z^{3}\right), \\
1-A^{2} \gamma(z)^{2} & =1-A^{2} z^{2}+O\left(z^{4}\right) .
\end{aligned}
$$

Using (3.8a) we get from (B.3) as $z \rightarrow \infty$

$$
\begin{aligned}
\gamma(z) & =-\frac{1}{z}+O\left(z^{-3}\right), \\
1-A^{2} \gamma(z)^{2} & =1-\frac{A^{2}}{z^{2}}+O\left(z^{-4}\right) .
\end{aligned}
$$

The function $\gamma(z)$ is piecewise analytic in $\zeta$ on the entire Riemann sphere, satisfying a jump condition at each $\zeta \in \mathbb{T}$. The only zeros of $\gamma(z)$ occur at zero and at infinity. Indeed, using (3.1) we see that $\zeta \sqrt{1+A^{2}}=z$ and $z^{-1}=\zeta^{-1} \sqrt{1+A^{2}}$ each imply that

$$
\frac{z-z^{-1}}{2}= \pm i \sqrt{1+A^{2}-\left(\frac{z+z^{-1}}{2}\right)^{2}} \text {. }
$$

However, the difference of the squares of the two sides of this equality equals $A^{2}$. Thus $\gamma(z)$ does not vanish if $0<|\zeta| \leq 1$ or if $1 \leq|\zeta|<+\infty$. 


\section{Appendix C. The Taylor series of $\gamma(z)$}

Let us write $z(\zeta)$ as a power series in $\zeta$ about $\zeta=0$. We get from

$$
z(\zeta)=\frac{\zeta+\zeta^{-1}}{2} \sqrt{1+A^{2}} \pm \sqrt{\left(\frac{\zeta+\zeta^{-1}}{2} \sqrt{1+A^{2}}\right)^{2}-1}
$$

with the square root defined such that the $\zeta^{-1}$ term about $\zeta=0$ drops out, that

$$
\begin{aligned}
z(\zeta) & =\frac{\sqrt{1+A^{2}}}{2 \zeta}\left\{1+\zeta^{2}-\sqrt{1-2 \frac{1-A^{2}}{1+A^{2}} \zeta^{2}+\zeta^{4}}\right\} \\
& =\frac{\sqrt{1+A^{2}}}{2 \zeta}\left\{\zeta^{2}-\sum_{l=1}^{\infty} \zeta^{2 l} \Pi_{l}\left(\frac{1-A^{2}}{1+A^{2}}\right)\right\},
\end{aligned}
$$

where

$$
\sqrt{1-2 B \xi+\xi^{2}}=\sum_{l=0}^{\infty} \xi^{l} \Pi_{l}(B)
$$

for $|\xi| \leq 1$ and $B \in[-1,1]$. Differentiating this series with respect to $\xi$ and $B$ and adding the resulting series, we obtain

$$
-\frac{1}{B} \sum_{l=0}^{\infty} \xi^{l}\left\{(l+1) \Pi_{l+1}(B)+\Pi_{l}^{\prime}(B)\right\}=\frac{1}{\sqrt{1-2 B \xi+\xi^{2}}} .
$$

Using the generating function of the Legendre polynomials, i.e.,

$$
\frac{1}{\sqrt{1-2 h \xi+\xi^{2}}}=\sum_{l=0}^{\infty} \xi^{l} P_{l}(h)
$$

for $|\xi|<1$ and $h \in[-1,1]$, we obtain

$$
(l+1) \Pi_{l+1}(B)=-\Pi_{l}^{\prime}(B)-B P_{l}(B) .
$$

Since $\Pi_{0}(B) \equiv 1$, we see that $\Pi_{l}(B)$ is a polynomial in $B$ of degree $l$. In fact, $\Pi_{0}(B)=1, \Pi_{1}(B)=$ $-B, \Pi_{2}(B)=\frac{1}{2}\left(1-B^{2}\right), \Pi_{3}(B)=-\frac{1}{2} B\left(B^{2}-1\right), \Pi_{4}(B)=-\frac{5}{8}\left(B^{2}-1\right)\left(B^{2}-\frac{1}{5}\right)$, etc. Also,

$$
\sum_{j=0}^{\infty} \xi^{j} \frac{\Pi_{j+2}(B)}{1-B^{2}}=\frac{\sqrt{1-2 B \xi+\xi^{2}}-1+B \xi}{\left(1-B^{2}\right) \xi^{2}}=\frac{1}{\sqrt{1-2 B \xi+\xi^{2}}+1-B \xi} .
$$

Then $\Pi_{j}(B)=\Pi_{j+2}(B) /\left(1-B^{2}\right)$ are $j$-th degree polynomials of $B$ having the generating function

$$
\sum_{j=0}^{\infty} \xi^{j} \Pi_{j}(B)=\frac{1}{\sqrt{1-2 B \xi+\xi^{2}}+1-B \xi} .
$$

Let us compute $\Pi_{j}(B)$. Using the generating functions of the Legendre and first kind Chebyshev polynomials [7, (4.7.2) and (4.7.18)], we compute

$$
\frac{\sqrt{1-2 B \xi+\xi^{2}}-1+B \xi}{1-2 B \xi+\xi^{2}}=\sum_{l=2}^{\infty} \xi^{l}\left[P_{l}(B)-T_{l}(B)\right]
$$


where we have used that $P_{0}(B)=T_{0}(B)=1$ and $P_{1}(B)=T_{1}(B)=B$. Therefore,

$$
\begin{aligned}
& \sum_{j=0}^{\infty} \xi^{j} \Pi_{j}(B)=\frac{\sqrt{1-2 B \xi+\xi^{2}}-1+B \xi}{\xi^{2}\left(1-B^{2}\right)} \\
& =\left(1-2 B \xi+\xi^{2}\right) \sum_{j=0}^{\infty} \xi^{j} \frac{P_{j+2}(B)-T_{j+2}(B)}{1-B^{2}} \\
& =\sum_{j=0}^{\infty} \xi^{j}\left[\frac{P_{j+2}(B)-T_{j+2}(B)}{1-B^{2}}-2 B \frac{P_{j+1}(B)-T_{j+1}(B)}{1-B^{2}}+\frac{P_{j}(B)-T_{j}(B)}{1-B^{2}}\right] .
\end{aligned}
$$

Consequently,

$$
\Pi_{j}(B)=\frac{P_{j+2}(B)-T_{j+2}(B)}{1-B^{2}}-2 B \frac{P_{j+1}(B)-T_{j+1}(B)}{1-B^{2}}+\frac{P_{j}(B)-T_{j}(B)}{1-B^{2}} .
$$

Using the recurrence relations for the Legendre and the first kind Chebyshev polynomials [7, (4.7.4) and (4.7.15)], we obtain

$$
\boldsymbol{\Pi}_{j}(B)=\frac{P_{j}(B)-P_{j+2}(B)}{(2 j+3)\left(1-B^{2}\right)}
$$

Using the Rodrigues formula $P_{s}(B)=\left(2^{s} s !\right)^{-1}\left(\frac{d}{d s}\right)^{s}\left(B^{2}-1\right)^{s}$, we obtain in terms of Jacobi polynomials [7]

$$
\Pi_{j}(B)=\frac{1}{2^{j+1}(j+1) !\left(B^{2}-1\right)}\left(\frac{d}{d B}\right)^{j}\left(B^{2}-1\right)^{j+1}=\frac{1}{2(j+1)} P_{j}^{(1,1)}(B)
$$

as a result of the Rodrigues formula for the Jacobi polynomials.

Let us now compute the Taylor series coefficients of $\gamma(z)$. We get

$$
\gamma(z)=\frac{\zeta \sqrt{1+A^{2}}}{2 A^{2}}\left\{1+\sum_{j=0}^{\infty} \zeta^{2 j} \Pi_{j+1}\left(\frac{1-A^{2}}{1+A^{2}}\right)\right\}
$$

where $|\zeta|<1$ and $A>0$. As a result, writing $\gamma(z)=\sum_{\sigma=0}^{\infty} \zeta^{\sigma+1} \gamma_{l, \sigma}$, we get $\gamma_{l, \sigma}=0$ for odd $\sigma$, $\gamma_{l .0}=\left(1+A^{2}\right)^{-\frac{1}{2}}$, and

$$
\gamma_{l, 2 j}=\frac{\sqrt{1+A^{2}}}{2 A^{2}} \Pi_{j+1}\left(\frac{1-A^{2}}{1+A^{2}}\right), \quad j=1,2,3, \ldots
$$

It is then easily verified that

$$
\Gamma_{l}(w) \stackrel{\text { def }}{=} \sum_{k=0}^{\infty} \gamma_{l, 2 k} w^{2 k}=\left(1+A^{2}\right)^{-1 / 2}-\frac{2\left(1+A^{2}\right)^{-3 / 2} w^{2}}{\sqrt{1-2 \frac{1-A^{2}}{1+A^{2}} w^{2}+w^{4}}+1-\frac{1-A^{2}}{1+A^{2}} w^{2}} .
$$




\section{Appendix D. Details on time evolution}

Indeed, putting $\Psi_{n}^{\text {as }}(z)=\left(\boldsymbol{Z}+\boldsymbol{q}_{r}\right)^{n} W_{r}(k)$, we compute, using $\sigma_{3}\left(Z-Z^{-1}\right)=\left(z-z^{-1}\right) I_{2}$ when evaluating $T_{r}$,

$$
\begin{aligned}
\left(1+A^{2}\right)^{-\frac{n}{2}} & \left(\boldsymbol{T}_{r} \Psi_{n}^{\mathrm{as}}-\left[\Psi_{n}^{\mathrm{as}}\right]_{t}\right) \mathscr{Z}^{-n} \\
& =\left[-\frac{1}{2} i\left(z-z^{-1}\right)^{2} \sigma_{3}-i A^{2} \sigma_{3}-i\left(z-z^{-1}\right) \boldsymbol{q}_{r}\right]\left[I_{2}+\gamma(z) \sigma_{3} \boldsymbol{q}_{r}\right] \\
& -\gamma(z) \sigma_{3}\left(-2 i A^{2} \sigma_{3} \boldsymbol{q}_{r}\right) \\
& =\left[-\frac{1}{2} i\left(z-z^{-1}\right)^{2}-i A^{2}-i\left(z-z^{-1}\right) A^{2} \gamma(z)\right] \sigma_{3} \\
& +\left[\left\{-\frac{1}{2} i\left(z-z^{-1}\right)^{2}+i A^{2}\right\} \gamma(z)-i\left(z-z^{-1}\right)\right] \boldsymbol{q}_{r} \\
& =[\ldots]_{1} \sigma_{3}+[\ldots]_{2} \boldsymbol{q}_{r},
\end{aligned}
$$

where

$$
\begin{aligned}
{[\ldots]_{2}+\gamma(z)[\ldots]_{1} } & =-\frac{1}{2} i\left(z-z^{-1}\right)^{2} \gamma(z)+i A^{2} \gamma(z)-i\left(z-z^{-1}\right) \\
& -\frac{1}{2} i\left(z-z^{-1}\right)^{2} \gamma(z)-i A^{2} \gamma(z)-i\left(z-z^{-1}\right) A^{2} \gamma(z)^{2} \\
& =-i\left(z-z^{-1}\right)\left[1+\left(z-z^{-1}\right) \gamma(z)+A^{2} \gamma(z)^{2}\right] \\
& =-i\left(z-z^{-1}\right)\left[1+\left(z-z^{-1}\right) \gamma(z)+\left\{\zeta \sqrt{1+A^{2}}-z\right\} \gamma(z)\right] \\
& =-i\left(z-z^{-1}\right)\left[1+\gamma(z)\left\{\zeta \sqrt{1+A^{2}}-z^{-1}\right\}\right] \\
& =-i\left(z-z^{-1}\right)\left[1+\gamma(z) \frac{-1}{\gamma(z)}\right]=0 .
\end{aligned}
$$

Observe that (3.6) has been used at the last two equality signs. Hence,

$$
\left(1+A^{2}\right)^{-\frac{n}{2}}\left(\boldsymbol{T}_{r} \Psi_{n}^{\text {as }}-\left[\Psi_{n}^{\text {as }}\right]_{t}\right) \mathscr{Z}^{-n}=[\ldots]_{1}\left\{\sigma_{3}-\gamma(z) \boldsymbol{q}_{r}\right\}=[\ldots]_{1} W_{r}(z) \sigma_{3} .
$$

Consequently,

$$
\left[C_{\Psi}\right]_{t} C_{\Psi}^{-1}=[\ldots]_{1} \sigma_{3},
$$

as claimed in (7.4). The quantity $\left[C_{\Phi}\right]_{t} C_{\Phi}^{-1}$ is evaluated in the same way. 